\title{
A linearization account of either ... or constructions
}

\author{
Philip Hofmeister
}

Received: 14 March 2007 / Accepted: 3 March 2008 / Published online: 20 February 2010

(C) The Author(s) 2010. This article is published with open access at Springerlink.com

\begin{abstract}
To account for the linear freedom of either in disjunction constructions, I expand upon the focus-based account of den Dikken (2006). Word order constraints, in contrast to movement rules or base-generation constraints, provide the mechanism for explaining the distributional data. I argue that all positional variability exhibited by either ultimately derives from a licensing construction that enables either to be shuffled about disjunct-internally, yet simultaneously prevents either from entering into linear precedence relations with disjunct-external constituents. Restrictions on the surface realization of either result from a linear precedence rule ordering either before the contrastive focus, language particular constraints on word order, and general constraints on coordinate ellipsis. Overall, this analysis presents an account of either ... or constructions that introduces only a single linear precedence rule and a licensing construction for combining either with disjunctions to account for the data, relying on independently-motivated constraints to carry the rest of the analytical burden.
\end{abstract}

Keywords Disjunctions · Free word order · Linearization · Contrastive focus

\section{Introduction}

For a language that typically imposes rigid constraints on word order, English does allow certain constituents to freely "float" about. Among these elements are sentential adverbs (probably, undoubtedly, etc.) and certain manner and measure adverbs (Ernst 2002, 2004), but also so-called coordination markers like either. The wide range of positions available to either is illustrated in the examples below:

P. Hofmeister $(\bowtie)$

Center for Research in Language, University of California-San Diego, 9500 Gilman Dr., Dept. 0526,

La Jolla, CA 90293-0526, USA

e-mail: phofmeister@ucsd.edu 
(1) $\langle$ Either $\rangle$ Thomas $\langle$ either $\rangle$ will $\langle$ either $\rangle$ write $\langle$ either $\rangle$ a mystery or he'll write a romance.

(2) $\langle$ Either $\rangle$ You'll $\langle$ either $\rangle$ need to $\langle$ either $\rangle$ bring $\langle$ either $\rangle$ a passport or a birth certificate.

(3) $\langle$ Either $\rangle$ Congress $\langle$ either $\rangle$ will $\langle$ either $\rangle$ pass the legislation or lose our confidence.

(4) $\langle$ Either $\rangle$ You $\langle$ either $\rangle$ can $\langle$ either $\rangle$ have $\langle$ either $\rangle$ tea $\langle$ either $\rangle$ from $\langle$ either $\rangle$ China or from Tibet.

Hence, either can mark disjunctions at the sentential, nominal, verbal, and prepositional level, not to mention disjunctions of complement clauses, adjectival phrases, and adverbial phrases:

(5) 〈Either $\rangle$ Thomas $\langle$ either $\rangle$ said 〈either $\rangle$ that he'll have coffee or that he'll have tea.

(6) The modem is 〈either $\rangle$ a small $\langle$ either $\rangle$ white or black box.

(7) You 〈either $\rangle$ can $\langle$ either $\rangle$ work $\langle$ either $\rangle$ quickly or slowly, but you must be thorough.

As is evident from the examples above, either can legitimately occupy different positions in and around the disjunction. There are, however, certain positions where either cannot appear:

(8) Thomas will read 〈*either〉 a book or take a nap.

(9) Thomas will read a $\langle *$ either $\rangle$ book or take a nap.

(10) Thomas will read a book $\langle *$ either $\rangle$ or take a nap.

(11) Thomas gave Angela 〈*either〉 the book or Marie (the book).

(12) Walter did not $\langle *$ either $\rangle$ remove his shoes or the dog tracked in this mud.

Either cannot surface between a verb and its object in a VP disjunction (8), or between a noun and its specifier (9), adjacent to or (10), after the first NP in a double object construction when only the first NP is contrasted (11), or under the scope of negative operators in clausal disjunctions (12). Other conditions and constructions further limit the positions available to either. Clearly, then, the surface position of either is not entirely unregulated.

This linear freedom, albeit limited, creates a problem for traditional syntactic assumptions about coordination and about the nature of coordination markers like $e i$ ther. On one hand, the symmetry condition on coordination (otherwise known as the Law of Coordination of Likes) states that phrases of different linguistic kinds cannot be coordinated, e.g. NPs and VPs (Chomsky 1957: 36). On the other hand, the leftedge hypothesis claims that markers like either sit at the left edge of the disjunctions they mark (Larson 1985; Sag et al. 1985). One of these conditions, however, would seem to be wrong in light of the data above. In (1), if two clauses or IPs are coordinated, then the later positions of either violate the left-edge hypothesis, i.e. either is 
lower than expected. If the left-edge hypothesis is maintained, however, then different syntactic categories are coordinated, violating the symmetry condition. Considering the earlier positions of either in (2), either unlike categories are coordinated, e.g. VP and NP, or either is not adjacent to the left edge of the disjunction, i.e. it is higher than expected. More generally, the problem is how to explain the wide range of positions open to this coordination marker and other floating elements, but at the same time explain why they can't show up in other positions. Ideally, these facts should have a unified account that ties together the licensed and unlicensed orderings.

These points have been well-observed in the syntax literature with as many explanations as there are discussions of the topic (Larson 1985; Schwarz 1999; Hendriks 2004; den Dikken 2006). The puzzle has been tackled with theories based on movement, ellipsis, and most recently, focus. In the latter case, den Dikken proposes that the positions available to either are largely determined by contrastive focus, following suggestions from Hendriks $(2001,2003)$. The explanatory power of this analysis stems from its handling of the "too low" and "too high" problems as being different aspects of the same problem, rather than treating them separately as previous analyses have done. The implementation of this theory, however, requires lengthy conditional statements about where either can appear along with an analysis of phrasal movement of $o r$.

Here, I expand upon, but essentially simplify, the focus-based approach to either ... or constructions by relying on linear precedence rules and constraints on word order shuffling. To develop this analysis, I draw on the word order domain work of Reape (1990, 1996), Kathol (2000), and a variant of the coordinate ellipsis approach used in Beavers and Sag (2004). In short, I propose that the positional variability exhibited by either can be explained by (i) coordinate ellipsis; (ii) a licensing construction that enables either to be shuffled about disjunct-internally, yet simultaneously prevents either from entering into linear precedence relations with disjunctexternal constituents; and (iii) a linear precedence rule (LP) ordering either before the contrastive focus. Only the last of these pertains specifically to either. Hence, this analysis introduces little more than a single linear precedence rule, depending on constraints otherwise necessary to explain facts about word order variation. In fact, even this single linear precedence rule may generalize to other types of "floating" elements such as adverbials, thereby reflecting a more basic fact about constituent ordering with respect to contrastively focused phrases. ${ }^{1}$ The guiding intuition is simple: because the function of either is to mark exclusive alternatives in a disjunction, it needs to precede the contrastive focus to do its job. This LP rule and the rule constraining the combination of either with disjunctions handle a majority of the distributional facts. The remainder is captured by word order constraints necessary for English, in general, and constraints on ellipsis in coordination structures.

In discussing the linearization tools necessary to handle the problem of either ... or, I will be relying on a theory of constructions, viz. that developed in Sag et al.

\footnotetext{
${ }^{1}$ The term "floating" is used in this paper to describe constituents that show positional variability due to domain merging, as described subsequently. Den Dikken (2006) uses this term in a decidedly different way to reflect the possibility of base-generating either in different syntactic positions.
} 
(2003: Chap. 16). In this theory, constructions ( $c x)$ act as constraints on local tree configurations: "a construction functions much like a context-free grammar rule" (Donohue and Sag 2004: 8). Essentially, these constructional constraints specify necessary relationships between syntactic mother nodes and a list of daughters. Constructions thus license only well-formed local trees or "constructs" ( $c x t)$. Thus, a constructional rule like "x-cxt $\Rightarrow[\ldots]$ " can be read as: "Members of a particular construct class (must) have the specific properties indicated" (Sag 2010: 2).

In the following section, I review the basics of den Dikken's (2006) analysis, showing the major strengths of the focus-based approach. Subsequently, I present empirical evidence that suggests that many word order positions for either previously labeled ungrammatical are, in fact, grammatical, although arguably less acceptable than other linearizations. In Sect. 4, I cover the recent advances in word order theory and, in the following section, illustrate how these linearization tools can be combined with the focus theory to account for the data with a bare minimum of additional stipulations. I end with a brief discussion of the import of linearization analyses for relatively fixed word order languages like English.

\section{Focus-based approach to either ... or constructions}

To provide a unified account of the distributional properties of either, den Dikken (2006) analyzes either as a phrasal category with a surface distribution dependent on contrastive focus. This analysis stands in contraposition to previous analyses (Larson 1985; Schwarz 1999) that only cope with either being "too high" as in (2) above. Schwarz (1999), for instance, argues that gapping accounts for the locality conditions on either, as illustrated below:

(13) John ate either [[NP rice] or [NP beans]].

(14) John either [vp ate rice] or [vp beans].

(15) Either [[IP John ate rice] or [IP John ate beans]].

On this view, the left edge of the disjunctive phrase is always marked by either, but certain constituents of the right disjunct are deletable under identity with constituents in the left disjunct. Gapping, however, cannot explain the grammaticality of (16) or analogous examples where either looks lower than expected.

(16) Thomas 〈either $\rangle$ will 〈either $\rangle$ write 〈either $\rangle$ a mystery or he'll write a romance.

In addition, both movement-based approaches and unrestricted gapping accounts predict the grammaticality of examples like (17), where either looks to have floated leftward across a clause boundary (all judgments are from den Dikken in this section):

$\langle *$ Either $\rangle$ John $\langle *$ either $\rangle$ said that he would eat rice or beans.

Den Dikken argues, however, that either cannot float upwards past a finite or nonfinite clause boundary.

In attempting to find a common explanation for both sets of facts, den Dikken (2006) highlights the connection between either and focus. This follows Hendriks 
(2001, 2003), who argues that either must c-command a contrastive focus. It is the interpretive scope of the contrastive focus that is identified as limiting the positions available to either, rather than just the location of the pitch-accented focus (capitalized words indicate the intonational focus; the interpretive scope is underlined):

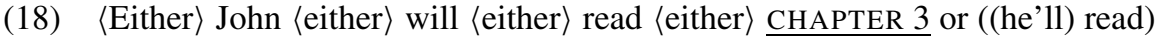
CHAPTER 4.

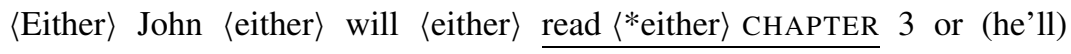
prepare DINNER.

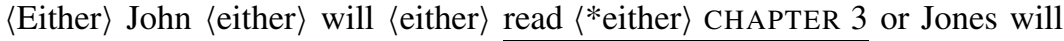
FLUNK him.

In each of these examples, the focus is marked intonationally only on the direct object, yet the interpretive scope of the contrastive focus extends beyond the direct object in the last two examples. The ungrammaticality of placing either directly adjacent to the direct object in these examples can therefore be interpreted as a byproduct of placing either inside the scope of the contrastive focus. The highest positions of either in (20) are argued to be available because contrastive focus is limited to the $v \mathrm{P}$; hence, it does not include the auxiliary and subject.

Although there is no formal description of how the scope of contrastive focus should be computed, it is suggested in the text (den Dikken 2006:716-717) that (i) an either ... or construction may theoretically have more than one possible contrastive scope assignment and that (ii) the scope possibilities can be assessed by determining what kind of statements the either ... or construction can felicitously follow up. For instance, (19) can follow the statement that John will do one of two things, but not John will read one of two things. Hence, contrastive focus can be at the VP level here, but cannot be restricted to the object NP. Observe, however, that (19) can also follow a more general statement, such as One of two things will happen; however, since focus is restricted to the $v \mathrm{P}$, the scope of contrastive focus will be the same. In contrast, all variants of (18) are felicitous continuations to John will read one of two things. After a statement such as John will do one of two things, however, contrastive focus will extend to the verb, causing the lowest position of either to be infelicitous, e.g. \#John will read either chapter 3 or read chapter $4 .^{2}$ Thus, the scope of contrastive focus will not be uniquely identifiable in some decontextualized examples; however, since disjunctions present contrasting focus alternatives, we can often determine the minimum extent of focus by simply identifying the structurally highest point where the disjuncts differ lexically. ${ }^{3}$

\footnotetext{
${ }^{2}$ Judgments of the infelicity of such a discourse sequence may vary here. Note that this position of either is possible under different preceding discourse conditions. The availability of at least some discourse context that could license the position of either appears to make the violations less dramatic. That is, the critical either-sentence is not by itself ruled out, but only in coordination with the preceding discourse. Moreover, in this particular example, where the type of action or event denoted in both disjuncts is the same, it is more natural to identify the contrastive focus as being limited to the direct objects. If the relevant events are taken as truly contrasting types of events, however, then the infelicity judgment associated with the low position of either should be sharpened.

${ }^{3}$ One exception, of course, is in clause-level disjunctions, where the focus will not include subjects and auxiliaries. The absence of lexical identity, however, will not be a perfect indicator of contrastive focus,
} 
Crucially, the scope of contrastive focus is distinguished from information focus (old-new information). The latter is typically assessed via the question-answer test (Selkirk 1995: 553): "A wh-question expression focuses a constituent, and an appropriate answer to a wh-question must focus the same constituent."

(21) Q: I thought John would read chapter 2, but somehow he didn't. What did he do instead?

A: $\langle$ Either $\rangle$ he $\langle$ either $\rangle$ read $\langle$ either $\rangle$ CHAPTER 3 or ((he) read) CHAPTER 4.

Information focus (single-underlining) must project up to the VP in this example, according to the question-answer test, yet either is acceptable within this scope. Contrastive focus (double-underlining), however, can be restricted to the direct object (but other assignments are possible, given a full VP or IP in the right-hand disjunct), as evidenced by the fact that the verb in the second disjunct is omittable. Notice that the variant of the answer with either between the verb and object is infelicitous following the statement that John did one of two things. ${ }^{4}$ The conclusion that is reached, therefore, is that either is sensitive to contrastive focus, but not information focus.

Contrastive focus by itself, however, seems insufficient to account for the full range of potential orderings, in the view of den Dikken. At a minimum, ccommanding just any contrastive focus does not look to be the appropriate desideratum, as either c-commands the contrastively focused object phrase, but not the contrastively focused subject NP in the following example.

\section{$\underline{\text { JOHN }}\langle *$ either $\rangle$ will $\langle *$ either $\rangle$ read $\langle *$ either $\rangle \underline{\text { CHAPTER } 3}$ or MARY CHAPTER 4.}

Yet the stronger constraint that either must c-command all contrastively focused constituents is not sufficient to account for the markedness of examples like (23), where either c-commands all foci:

$\langle$ ??Either $\rangle$ He $\langle$ ??either $\rangle$ said $\langle \%$ either $\rangle$ that he would eat RICE or BEANS.

Moreover, either apparently does not always have to precede all contrastive foci in a sentence, as illustrated in the following question-answer pair:

Q: Did John say that he had either FRIED it or BAKED it?

A: No! John $\langle *$ either $\rangle$ DENIED $\langle *$ either $\rangle$ that $\langle *$ either $\rangle$ he $\langle *$ either $\rangle$ had $\langle$ either $\rangle$ fried it or baked it.

The relevant contrastive focus in the answer occurs in the matrix clause (i.e. at denied). Yet the only indicated acceptable position for either given in the example does not c-command this focus. Assuming that this sentence reflects a disjunction over

e.g. Sawyer will have a sandwich or he'll take a salad. This sentence can follow up Sawyer will have one of two things for lunch, restricting contrastive focus to the direct object, even though the verbs differ. The key, therefore, is that the contrastive focus alternatives must be felicitous arguments for the non-contrasted predicates in each disjunct.

${ }^{4}$ Certain linear orderings of either, therefore, will technically be infelicitous given the preceding discourse, although such an ordering may be perfectly acceptable in other contexts. In other cases, the position of either is unacceptable regardless of preceding context, resulting in a strong perception of unacceptability. 
VPs, the only legitimate position for either here appears to be at the left edge of the disjunction. On the basis of this data, the conclusion is reached that, in certain contexts, "either's distribution is entirely insensitive to the locus of contrastive focus, and is subject solely to the requirement that either be placed on the first disjunct" (p. 706).

Cumulatively, these data lead den Dikken to the following generalization about the distribution of either (p. 707), where "attach" here means the base-generation point:

(25) either is a phrasal constituent in construction with

a. the first disjunct, attaching to it; or

b. the first contrastive focus, attaching to

i. the contrastive focus itself, or

ii. a phrasal node on the $\theta$-path projected from the first contrastive focus

Here, a $\theta$-path is defined as a sequence of nodes that are $\theta$-linked, i.e. one node assigns a $\theta$-role to the other. By way of this, a $\theta$-path can be made from one phrase to a higher phrase if the head of the latter assigns an internal $\theta$-role to the former. Accordingly, a $\theta$-path cannot project from an IP to a $\mathrm{CP}$, given that $\mathrm{C}$ does not have a thematic relationship with IP, e.g. neither assigns a $\theta$-role to the other. This accounts for the markedness of examples like (23), because either is not on a $\theta$-path projected from the contrastively focused direct object, i.e. CP disrupts the $\theta$-path. Similarly, the bad cases of either in (19)-(22) are accounted for. In (19) \& (20), either shows up inside the indicated scope of contrastive focus-hence, not along the $\theta$-path or adjacent to the focus or the first disjunct; in (22), either does not precede the first disjunct or the first contrastive focus.

Even with the conditions in (25), however, a number of facts still remain unaccounted for. Among these is the inability of either to surface under negation in IPlevel disjunctions:

\section{*John didn't eat either RICE or he didn't eat BEANS.}

Technically, the conditions listed above sanction this ordering (either is attached to the first contrastive focus), but negation seems to complicate the picture. In response, it is claimed that the fault lies with or, leading to an "analysis of or as a phrasal category that moves to the left edge of the second disjunct whenever it does not originate there" (p. 734). In particular, den Dikken speculates that "or is preferably base-generated on the second disjunct" (p. 733), i.e. at the left edge, but if either is base-generated within the first disjunct " $o r$ 's base position in the second will match $e i$ ther's in the first" (p. 734). In other words, either and or start off in identical structural positions in each disjunct, and while either stays put, or participates in movement to get to the left edge of the disjunct. This movement requirement is motivated by the assumption that the (empty) functional head of the disjunction has a set of uninterpretable formal features that have to be checked against a matching set of features carried by $o r$.

The unacceptability of (26), on this analysis, is the result of the 'illegal' movement of or across sentential negation, which supposedly establishes an inner island. That is, or is base-generated adjacent to the contrastive focus in the right disjunct, 
matching the location of either in the left disjunct, but or must eventually move to the disjunct edge to establish checking relations. The sentential negation, however, putatively blocks this movement.

(27) [John didn't eat either RICE] or ${ }_{i}$ [he didn't eat $\left\langle{ }^{*} t_{i}\right\rangle$ BEANS].

Likewise, in (28), neither position of either is possible, despite satisfying the requirements in (25):

(28) John was [reading a book $\langle *$ either $\rangle$ about $\langle *$ either $\rangle$ CHOMSKY] or ${ }_{i}$ [reading a book $\left\langle * t_{i}\right\rangle$ about $\left\langle * t_{i}\right\rangle$ CHOPSTICKS].

On the account of or-generation and movement just sketched, however, or must be base-generated in the same structural position in the second disjunct as either occupies in the first disjunct. Base-generation inside the NP in the second disjunct, followed by movement of $o r$ to the left edge, requires movement out of a complex noun phrase. Although these extractions do not violate the complex noun phrase constraint or Subjacency (Ross 1967; Chomsky 1973), this movement-type is qualified as being impossible: "what we have on our hands is more like scrambling in languages like Dutch or German, which is known not to be able to transgress the boundaries of a complex noun phrase" (p. 737). This island sensitivity is thus taken as further evidence for a movement account of or, completing the picture of either ... or constructions.

This analysis of or-movement, though, raises the question of what ensures the symmetrical realization of either and or in the separate coordinates. It is asserted that "or originates in the same hierarchical position within the second disjunct as either does in the first" (p. 734), but disjuncts need not have the same hierarchical structure. In some cases, the surface position of either cannot be the guide for the base-generation for $o r$, because comparable hierarchical positions do not clearly exist in the right disjunct:

(29) Puree in either a food processor or I use a hand blender.

[livinlavidalocarb.blogspot.com/2006/01/long-term-atkins-weight-loss-is.html]

... and then add a layer of olive oil to the pan with either a towel (or I use a pump spray).

[chowhound.chow.com/topics/353666]

Additionally, there is no available explanation for why the presence of either should interfere with the stated preference that $o r$ should be base-generated at the edge of the disjunct. There is no evident justification of this parallelism constraint, nor a way it could be implemented formally, yet a serious computational problem seems to emerge if the base-generation point of one constituent determines the generation point of some second constituent that can be arbitrarily far away.

It is also worth noting that for the $\theta$-path analysis to work for a variety of examples requires the assumption that I assigns a $\theta$-role to VP:

$\langle$ Either $\rangle$ He $\langle$ either $\rangle$ would read a BOOK or a MAGAZINE. 
This assumption is justified by claiming (following Chomsky 1986) that the following $w h$-island example is only mildly degraded, implying ECP satisfaction and hence proper government of the VP trace by I:

\section{? [VP Fix the car $]_{i}, I$ wonder $\left[\mathrm{CP}\right.$ whether [IP he will $\left.\left.\mathrm{t}_{\mathrm{i}}\right]\right]$}

It is difficult to assess the sufficiency of this evidence, because of its acknowledged markedness and the lack of any more convincing examples. Additionally, this $\theta$ path analysis critically requires adverbial, adjectival, and prepositional modifiers to receive $\theta$-roles from their modifiees - a highly non-standard assumption about $\theta$ linking that conflicts with the definitional use of $\theta$-role. The reasoning used to support this assumption is rather circular: the fact that either can be left-displaced from a contrastively focused modifier is used to "show that $\theta$-paths can be projected from constituents that are syntactically adjuncts” (p. 711), as in (33):

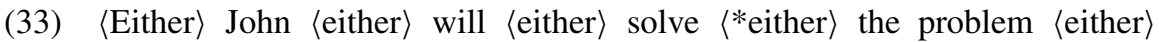
QUICKLY or (he will solve the problem) THOROUGHLY.

An alternative interpretation of this data, though, is that the $\theta$-path analysis is incapable of fully accounting for the distribution of either.

Finally, the distributional generalization in (25) states two seemingly unrelated conditions. It is not transparent why either should be sensitive to the location of focus in some contexts, but "entirely insensitive" in others. While nothing in theory prevents an analysis where unrelated constraints account for the distribution of some item, disjunctive generalizations of natural and systematic phenomena ideally express alternatives that each satisfy some common set of conditions or criteria, albeit in differ ways. In the case at hand, it is presently unclear what common requirements the alternatives in (25) both fulfill.

The focus-based approach, therefore, has many pluses in its favor, but the particular instantiation leaves some unanswered questions and requires some unrelated assertions. As we shall see, there are also some empirical gaps in this account. The evidence is nevertheless overwhelming that contrastive focus must be incorporated into any analysis of the distribution of either. Most significant is the fact that either cannot float down inside the interpretive scope of the contrastive focus. This observation therefore constitutes the centerpiece of my own analysis. Before I present the details of my analysis, though, I turn first to a consideration of some empirical data on the word order possibilities for either.

\section{Evidence for a freer either}

Den Dikken and other researchers writing on the subject of either ... or constructions have supplied a wealth of judgments and data on various word order possibilities, the bulk of which stem from the authors' intuitions (or informal polling of other linguists). These judgments have been characterized by a range of grammaticality diacritics composed of various combinations of stars, question marks, percentage signs, and even exclamation marks. Den Dikken, for instance, employs four different diacritics $(?, ? ?, \%, *)$ without laying out any criteria for how such assignments are made 
or any theoretical commitments to these different labels. On the basis of this range of categorizations alone, it should be evident that there are subtleties of judgment involved and therefore not surprising that authors on the subject sometimes disagree with one another about grammaticality judgments.

Instead of simply providing my own intuitions on the legitimate positions of $e i$ ther, I rely on empirical evidence based on simple internet searches and corpus work to confirm whether particular either ... or tokens constitute acceptable constructions of English. While the mere existence of one or two tokens of a particular word order cannot provide incontrovertible proof of grammaticality, hundreds of such examples should warrant the conclusion that the word order is acceptable under at least some conditions (particularly when such examples appear in texts that have almost certainly been edited to some degree). ${ }^{5}$ Moreover, contextualized examples often sound surpassingly better than constructed and decontextualized examples and can sometimes reveal the reasons for the degradedness of the out-of-context examples.

For attested examples, however, there is the issue of how to determine the scope of contrastive focus, since this plays a critical role in the present analysis. I will follow den Dikken in claiming that some sentences may have more than one possible focus assignment. But on the straightforward assumption that elements of the contrastive focus cannot be deleted (Heim 1997; Romero and Han 2003)-elision of interpretively contrasted material would impair computation of the focus-the contrastive focus can safely be estimated as being no larger than the surface form of any disjunct. For instance, if the right-hand disjunct only contains an adverb as in (34) below, contrastive focus will necessarily be restricted to the adverbial level in both disjuncts. But for larger constituents, focus can extend higher, e.g. up to the VP level in (35). Any disjunction structure also provides clues to the extent of the interpretive focus, since the disjunction lists a set of alternatives that must presumably be at the same syntactic level (NP, VP, PP, etc.). Moreover, each alternative must be compatible with the non-focused predicates in each disjunct. Hence, even though chapter 3 and dinner are theoretically possible alternatives, they are not plausible arguments of both verbs in John will read chapter 3 or prepare dinner. Consequently, the interpretive focus clearly must extend beyond the direct object. Note that we do find examples where it is impossible to identify a unique focus assignment, but the surface position of either is predicted to precede at least one possible contrastive focus assignment.

Turning to the data now, den Dikken claims that either cannot float upward past a clause boundary, finite or non-finite. This assertion guides the formulation of his solution and distinguishes his account from theories of either-placement based solely on gapping. Indeed, none of the relevant literature openly suggests that either can float upward beyond a clause boundary. Simple internet searches, however, quickly reveal numerous examples like the following:

\footnotetext{
${ }^{5}$ Unfortunately, it is impossible to provide accurate frequency counts of the different word order positions of either in disjunctions using the internet as the corpus, as structural information is unavailable, which makes searches dependent upon specific lexical choices. Furthermore, the data shown here were based on searches using wildcards, thus returning sizeable amounts of irrelevant data, which prevents estimations based on internet search counts. Tagged corpora, on the other hand, are too limited in size to provide the necessary information. The examples that follow are intended to largely provide proof of existence; however, for each word ordering, similar examples are not difficult to find.
} 
(34) Depending on those values, the equation may either suggest that life arises frequently or infrequently.

[http://en.wikipedia.org/wiki/Life]

(35) It can either be said that the inverter absorbs lagging current or provides leading current ...

[High Voltage Direct Current Transmission, by Jos Arrillaga, IET, 1998, p. 30.]

(36) Their subjects had to cheer as loudly as possible under conditions where subjects either believed that they cheered alone or as part of groups of different sizes.

[Understanding Group Behavior, by Erich Witte and James H. Davis, LEA, 1996, p. 40.]

(37) They either know that they owe tax or get a refund.

[http://www.citizenstaxrelief.com/income-tax-problems-article.php]

On the focus/base-generation account, such high positions should be ruled out because either does not appear along the $\theta$-path extending from the contrastively focused phrase inside the CP. Due to the sheer number of such attested tokens and the fact that such examples fail to exhibit any signs that they constitute performance errors (e.g. they appear in presumably edited texts and books), it appears that such constructions are actually grammatical, although they may be generally dispreferred for reasons independent of the grammar.

To evaluate the grammaticality of these "high" positions of either, an acceptability survey was constructed using tokens extracted from the internet, such as those above. The materials for this survey consisted of twenty such naturally-occurring examples with either appearing in one of four positions: above the matrix verb (HIGH), immediately after the complementizer (MID), in a position just to the left of a constituent whose phrasal type is identical to that of the overt right disjunct (LOW), or immediately preceding or (ERROR). Subjects saw each item in only one condition (Latin-square design), and were instructed to rate examples for naturalness on a scale of 1-7 with 7 being the most natural.

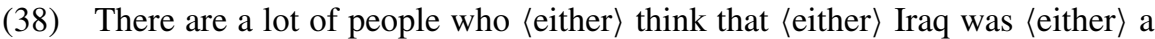
doable proposition that was botched $\langle$ either $\rangle$ or a project destined for failure.

The experimental items were paired with ninety-four filler items, among which were a set of twelve agreement violations. Twenty-four Stanford University undergraduates participated in the study for $\$ 14 /$ hour.

The acceptability results reveal that "high" positions of either are judged to be as good as the lower positions of either (all $t$-scores $<1$ ). In fact, there are no statistically reliable differences among the positions of either that are not adjacent to or $(\mathrm{HIGH}=6.15, \mathrm{SE}=.126 ; \mathrm{MEDIUM}=6.18, \mathrm{SE}=.108 ; \mathrm{LOW}=6.13, \mathrm{SE}=.122$; $\mathrm{ER}-$ $\mathrm{ROR}=3.62, \mathrm{SE}=.172$ ). Crucially, all of these higher positions were deemed significantly better than sentences with either adjacent to or, including the highest position of either above the matrix verb (HIGH-ERROR: $t_{1}(23)=8.615, p<.001, t_{2}(19)=$ $12.269, p<.001$; MEDIUM-ERROR, $t_{1}(23)=8.247, p<.001, t_{2}(19)=13.401$, 
Fig. 1 Mean acceptability scores for four positions of either and agreement violations (AGR-ERROR)

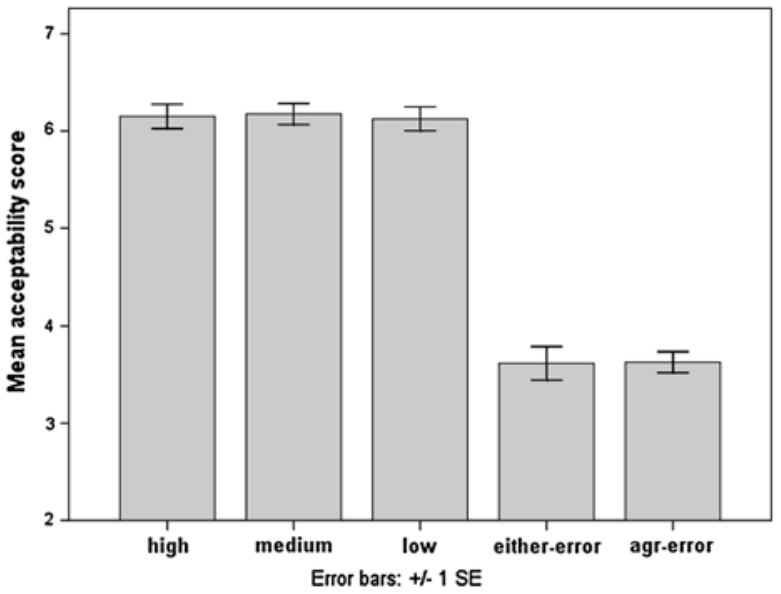

$p<.001$; LOW-ERROR, $\left.t_{1}(23)=8.192, p<.001, t_{2}(19)=14.368, p<.001\right){ }^{6}$ In other words, the highest positioning of either was evaluated as being significantly better than a clear word order error, but no different from incontrovertibly acceptable lower positions. As depicted in Fig. 1, the positions of either adjacent to or were judged on a par with agreement violations (AGR-ERROR in Fig. 1). Together with the naturally-occurring examples, this evidence strongly supports the conclusion that either can be separated from the contrastive focus by a clause boundary.

Den Dikken's focus-based account furthermore does not license unbalanced prepositional coordinations: "embedding either inside the complement PP is legitimate if and only if disjunction is at the level of P's complement ... as soon as the disjuncts are as large as PP or larger, either is prevented from occurring inside PP" (p. 735). This statement, however, conflicts with the existence of examples like the following that appear pervasively in all types of language media:

(39) Participants with backgrounds in either sociolinguistics or in experimental methods are welcome.

[http://ling.lsa.umich.edu/grad/courses.html]

(40) ... you may choose to subscribe to either DirecTV or to the Dish Network.

[http://www.sbgi.net/template/shared_content/timewarner/index.shtml]

(41) They cultured blood cells with either GFD or with a purified human antibody as a control.

[http://www3.niaid.nih.gov/news/newsreleases/2005/catdander.htm]

As evidence that floating deeply into a PP is possible, see (42), also found on-line:

\footnotetext{
${ }^{6}$ Even with Bonferroni corrections for multiple comparisons, the t-values still yield significance at the alpha-level of .001. 
(42) Send me an email to the three course addresses given on the Course Pages, with the answers either in the body of the email, or with the file sent as an attachment to the message.

[http://www.drshirley.org/helps/FAQ.html]

Arguably, these imbalanced coordinations appear less frequently than the superficially balanced disjunctions with either outside the PP, but the sort exemplified in (39)-(42) nevertheless occur in the thousands on a simple internet search.

Other data central to this discussion seem similarly questionable. In building an argument for the gapping account, Schwarz (1999) contrasts verb-particle constructions examples like those below. According to Schwarz, the particle must appear in the first disjunct and so examples like (43a) are usually classified as ungrammatical or highly deviant:

(43) a. Either he gulped one or two down.

b. Either he gulped down one or two.

Again, however, examples of the supposedly ungrammatical sort are not hard to find:

(44) Usually, this leaves the system designer to either start one or two channels up at a time.

[http://www.freepatentsonline.com/EP0855617.html]

(45) When we used the bathroom, we had to tell the staff in sign language (by either holding one or two fingers up), whether we had to urinate or defecate.

[http://cafety.org/index.php?option=com_content $\ \& t a s k=v i e w \backslash \& i d=488 \backslash \&$ Itemid=35]

Furthermore, to the extent that examples like (43a) are marked, this status appears to be independent of either, as the acceptability contrasts cited in the literature persist when either is absent.

Overall, this evidence suggests that either enjoys a freer positional variability than earlier thought. Other studies, including den Dikken (2006) and Han and Romero (2004), acknowledge the variability of judgments regarding constructions like these and other either ... or constructions. This awareness, though, is not concomitant with an explicit account of what this variability reflects or how it should be handled. In general, deviance of any kind has tended to push a given word order into the ungrammatical status. Unquestionably, certain word orders occur with less frequency than others and this frequency distribution appears to roughly correlate with how syntactic research has binned particular word orders into grammatical or ungrammatical. Low frequency, however, is not tantamount to ungrammaticality, as various performancerelated factors can cause a particular construction or word order to be dispreferred and therefore infrequent. For instance, while complex center-embeddings occur with an exceptional rarity, their unacceptability (and associated infrequency) has been linked to performance-related factors, rather than grammatical principles (Miller and Chomsky 1963). The same reasoning can be extended to the word order variability evident in either ... or constructions. Functional considerations may limit certain word orders from surfacing, despite their grammaticality. We may well imagine that either is 
typically preferred close to the superficial edge of the disjunction because this proximity facilitates the identification and interpretation of the disjunction. At present, however, my purpose is not to identify the performance-related factors that could account for the distributional frequency of word orders. Rather, it is merely to point out that functional or processing-related considerations can explain why certain word orders have been previously deemed ungrammatical in contrast with what the empirical evidence indicates. Based on this evidence, therefore, I treat word orders like those above as fully grammatical.

\section{Word order domains}

In transformational theories of syntax, hierarchical structure and linear order are codependent. Word order is determined by reading off the terminal yield of the syntax tree and bracketing or hierarchical structure is defined by the string sequence (Chomsky 1957, 1973). There is thus a general blurring of the distinction between hierarchical structure and linear order. Scrambling, free word order, and discontinuous constituency, however, are all phenomena that present serious challenges to standard theories of syntax that assume "structural hierarchy, although covert, is directly reflected in the sequence of words that constitute a sentence" (Pollard et al. 1994). ${ }^{7}$ The evidence of a dissociation between these structures necessitates characterizing linear order and constituency according to independent principles (e.g. Gazdar and Pullum 1981).

As one example of an attempt to define the independent constraints on linear order, Reape (1996) proposes that lexical and phrasal representations bear linear word order specifications, distinct from the level of representation that determines hierarchical relationships. In HPSG terms, linguistic signs are specified for a DOM(AIN) feature, which has a list of signs as its value. This is exemplified in the following featurestructure representation for signs, which also contains information about phonology (PHON), syntax (SYN), semantics (SEM), and morphological lemma form (FORM):

$$
\left[\begin{array}{l}
\text { sign } \\
\text { PHON phon } \\
\text { FORM list }(\text { form }) \\
\text { SYN }\left[\begin{array}{l}
\text { syn } \\
\text { HEAD ... }
\end{array}\right] \\
\text { SEM sem } \\
\text { DOM list }(\text { sign })
\end{array}\right]
$$

\footnotetext{
${ }^{7}$ As Pollard et al. (1994) notes, derivational theories of syntax avoid the general difficulties introduced by discontinuous constituents because the various stages of the derivation separate an underlying constituency from the surface string or word order.
} 
The elements on a DOM list correspond to hierarchical constituents and the order of the elements on the list determines the order of pronunciation (the value of the feature PHON), as expressed in the Constituent Ordering Principle: ${ }^{8}$

Constituent Ordering Principle

$$
c x t \Rightarrow \operatorname{MOTHER}\left[\begin{array}{l}
\operatorname{PHON} \phi_{1} \oplus, \ldots, \oplus \phi_{n} \\
\left.\operatorname{DOM}\left\langle\left[\operatorname{PHON} \phi_{1}\right], \ldots,\left[\operatorname{PHON} \phi_{n}\right]\right\rangle\right]
\end{array}\right]
$$

This principle says that each non-terminal syntactic node (e.g. syntactic M(O)T(HE)R) is specified for word order domains, and this value is used to construct the PHON value. It is thus at the level of the DOM list that language-specific linear precedence rules, like those in (48), are enforced:

$$
\begin{aligned}
& \text { Sample Linear Precedence Rules for English } \\
& \text { LP1: DET } \prec \text { NOUN } \\
& \text { LP2: AUX } \prec \text { VERB }
\end{aligned}
$$

Linear precedence relations within a word order domain are not subject to change even as larger word order domains are built from smaller domains (Kathol and Pollard 1995; Yatabe 2001):

\section{Persistence Constraint}

Any precedence relations holding of domain objects in one order domain are also required to hold of those objects in all other order domains that they are members of.

These word order domain lists are built up systematically on the basis of the domain values of syntactic daughters. For a fixed word order language like English, the DOM list for each syntactic mother node typically contains one element corresponding to each structural daughter. These constraints are expressed in the specifications for "concatenating" constructions, where linear order corresponds with the order of syntactic combinations (' $\mathrm{A} \oplus \mathrm{B}$ ' means the list composed of $\mathrm{A}$ and $\mathrm{B}$ where all elements of A precede all elements of B):

$$
\text { concat } \text { c } x t \Rightarrow\left[\begin{array}{l}
\text { MOTHER }\left[\mathrm{DOM} \delta_{1} \oplus \delta_{2}\right] \\
\operatorname{DTRS}\left(\left[\operatorname{DOM} \delta_{1}\right],\left[\operatorname{DOM} \delta_{2}\right]\right)
\end{array}\right]
$$

To provide a simple example of how this works, consider the English sentence, I can't dance, with the following simplified feature-value representations (e.g. the SYN value abbreviates the appropriate syntactic category and associated feature-value specifications):

\footnotetext{
${ }^{8}$ Here, and in all subsequent AVMs (attribute-value matrices), angled brackets denote a list, while square brackets contain feature-value pairs. Hence, a list may be composed of a set of feature-value pairs, as in the value specification for DOM in (47). Small caps signify a feature label.
} 


$$
\begin{aligned}
& {\left[\begin{array}{l}
\operatorname{PHON}\langle I, \text { can't, dance }\rangle \\
\operatorname{DOM}\langle[\mathrm{I}]\rangle \oplus\langle[\text { can't }]\rangle \oplus\langle[\text { dance }]\rangle \\
\operatorname{SYN} S
\end{array}\right]} \\
& {\left[\begin{array}{l}
\text { PHON }\langle I\rangle \\
\text { DOM }\langle[\mathrm{I}]\rangle \\
\text { SYN NP }
\end{array}\right] \quad\left[\begin{array}{l}
\text { PHON }\langle\text { can't, dance }\rangle \\
\text { DOM }\langle[\text { can't }]\rangle \oplus\langle[\text { dance }]\rangle \\
\text { SYN VP }
\end{array}\right]} \\
& {\left[\begin{array}{l}
\text { PHON }\langle\text { can't }\rangle \\
\text { DOM }\langle[\text { can't }]\rangle \\
\text { SYN } A U X
\end{array}\right]\left[\begin{array}{l}
\text { PHON }\langle\text { dance }\rangle \\
\text { DOM }\langle[\text { dance }]\rangle \\
\text { SYN VERB }
\end{array}\right]}
\end{aligned}
$$

In the case of the terminal nodes here, the singleton DOM values pass directly to the PHON value. The DOM values of the VP and S nodes, in turn, simply comprise the concatenation of their daughters' DOM values. In this basic example, therefore, concatenation preserves the hierarchical structure in the linear order of the constituents.

Crucially, the word order theory of Reape (1996) and the expanded account in Kathol (2000) also permit dissociation between the hierarchical and linear orders. What enables this dissociation is the availability of another type of operation (besides concatenation) on daughter DOM lists: domain merging. Unlike concatenation, domain merging allows elements on two DOM lists to intermingle, much like shuffling two stacks of cards together. The process of merging two DOM values (represented with the symbol ' $\bigcirc$ ') preserves the respective order among elements of the original domains, but allows intermingling of elements from distinct word order domains. For instance, merging the two sets $\langle a, b\rangle$ and $\langle c, d\rangle$ yields six different orderings, but in all cases, $a$ precedes $b$ and $c$ precedes $d$ (ex. from Kathol 2000).

$$
\langle a, b\rangle \bigcirc\langle c, d\rangle \in\left\{\begin{array}{l}
\langle a, b, c, d\rangle,\langle a, c, b, d\rangle,\langle a, c, d, b\rangle \\
\langle c, a, b, d\rangle,\langle c, d, a, b\rangle,\langle c, a, d, b\rangle
\end{array}\right\}
$$

This operation, along with the inheritance of domain lists, allows elements of two domain lists that belong to non-sister constituents to enter into linear precedence relations, creating potential word order variability. Constructs (i.e. local trees) that preserve the number of domain elements on the daughters' list on the mother's list, but allow intermingling of those domain elements, obey the constraints for so-called "liberating" constructions (Donohue and Sag 2004):

$$
\text { liberating-cxt } \Rightarrow\left[\begin{array}{l}
\text { MOTHER }\left[\operatorname{DOM} \delta_{1} \bigcirc \ldots \bigcirc \delta_{i}\right] \\
\operatorname{DTRS}\left\langle\left[\operatorname{DOM} \delta_{1}\right], \ldots,\left[\operatorname{DOM} \delta_{i}\right]\right)
\end{array}\right]
$$

(53) says that the mother's domain value in a liberating construct is a list of domain elements made up of all the elements of the daughters' domain lists. Hence, if each 
daughter has two elements on its domain list, the mother's list will have four. The final order of these elements, however, is not determined by (53), but by LP rules in the given language. If LP rules do not force one specific order, then multiple word order possibilities are licensed.

By way of illustration, consider the case of a sentential adverb like unfortunately, whose surface position can legitimately vary in English (Ernst 2002). Assuming that the constructional rule that licenses the combination of a sentential adverb and verb phrase is a type of a liberating construction, the constituent ordering principle in (47) and the liberating construction rule in (53) predict the ordering possibilities for the adverb in the following example:

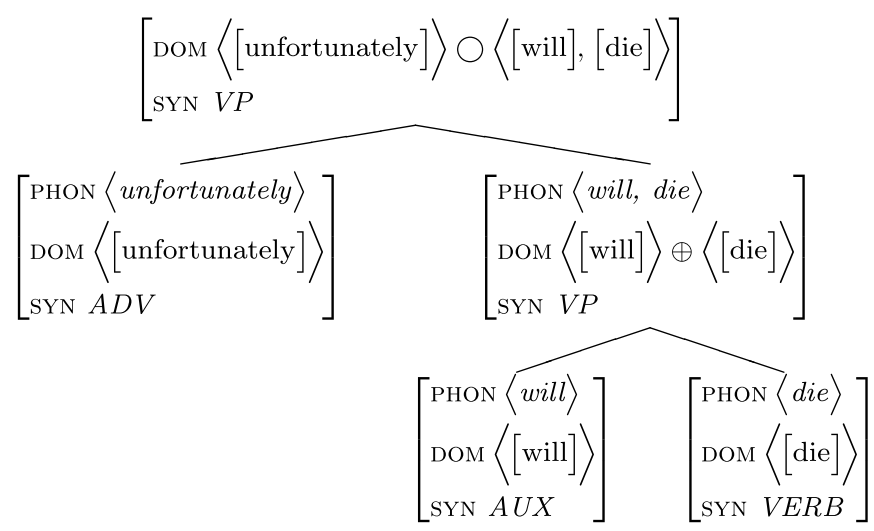

The domain value of the lower VP node is formed via concatenation as before, but the liberating construction rule licenses the topmost VP node here, as its DOM value is defined as the merging together of its daughters' DOM lists. The result is a list with three members. Since will and die were previously concatenated, their relative order must remain the same, but nothing constrains the positioning of unfortunately: while concatenation preserves the relative order of constituents, it does not prevent those constituents from being separated. As expected, then, three possibilities are available for the value of PHON in the topmost node: $\langle$ unfortunately, will, die $\rangle$; $\langle$ will, unfortunately, die $\rangle$; 〈will, die, unfortunately $\rangle$.

In such a manner, non-sister constituents can be elements of the same word order domain. The merging effectively produces a set of sister constituents over which linear precedence rules can apply, although hierarchically-speaking, the relevant constituents need not be syntactic sisters. As Donohue and Sag (2004) note, "[t]his creates the possibility of second or third generation scrambling-i.e. first or second cousins interspersing with one another on their grandmother's domain list" (p. 8).

Some syntactic phrases, however, restrict any other words or phrases from intermingling with their elements (Dowty 1995). Noun phrases, for instance, do not tolerate the adverbs already seen to float about. Hence, undoubtedly cannot appear between the specifier and head noun in (55), while the heavy noun phrase in (56) cannot contain probably when it is used to modify the main clause verb: 
*The undoubtedly patient will die.

(56) *His decision to run probably for president will annoy some people.

This can be modeled by specifying that the elements on the DOM list of the NP cannot enter into linear relations with elements of any other word order domain. Formally, such a constraint is introduced in the specifications for "compacting" constructions, where word order elements are pressed together into a single DOM element (Chaves 2007; Donohue and Sag 2004):

$$
\text { compacting-cxt } \Rightarrow\left[\begin{array}{l}
\text { MOTHER }\left[\operatorname{DOM}\left\langle\left[\operatorname{DOM} \delta_{1} \bigcirc \ldots \bigcirc \delta_{i}\right]\right\rangle\right] \\
\left.\operatorname{DTRS}\left\langle\left[\operatorname{DOM} \delta_{1}\right], \ldots,\left[\operatorname{DOM} \delta_{i}\right]\right\rangle\right)
\end{array}\right]
$$

The crucial difference between (53) and (57) is that liberating constructions require the cardinality of the mother's DOM list to match the number of elements on the daughters' lists, but a compacting construction licenses only one element on the mother's DOM list and this element is a list composed of the daughters' domain elements. To illustrate, if $a$ must precede $b$, the compaction of the singleton lists $\langle a\rangle$ and $\langle\mathrm{b}\rangle$ will result in the new singleton list, $\langle[\langle a, b\rangle]\rangle$ (equivalently written as $\langle[a, b]\rangle$ ). Once compacted, elements in a word order domain cannot intermingle with elements from any other list. Essentially, compaction shuts off a linear domain from outside interference. Domain internally, however, word order remains free, since the domain elements are shuffled together.

To see how this works, consider (58) below:

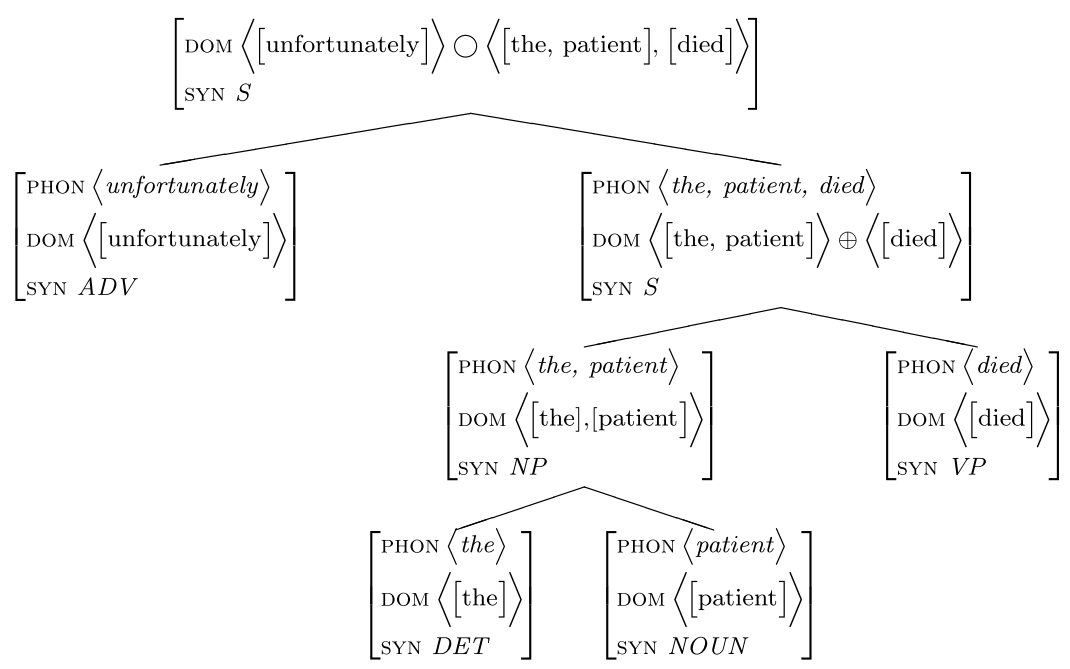

On the assumption that the combination of NPs with their heads is licensed by a type of compacting construction, i.e. nominally-headed specifiers and complements 
compact when combined with their heads (Chaves 2007), the word order domain of the NP node will compact at the lower S node, as represented by the single element in brackets in the DOM list. Because of this, even after the $\mathrm{S}$ domain gets shuffled with another domain element, unfortunately, the determiner and noun remain inseparable. Ultimately, the top node leads to only three possible orderings: 〈unfortunately, the, patient, died $\rangle$; $\langle$ the patient, unfortunately, died $\rangle$; $\langle$ The, patient, died, unfortunately $\rangle$.

The linearization tools thus provide a way of modeling free word order via domain shuffling, but domain compaction and linear precedence rules moderate this freedom. While the merging operation is a powerful tool for realizing different word order possibilities, it is thus limited in practice by the combination of the persistence constraint, LP rules, and instantiations of compaction. For a language like English with relatively fixed word order, this means that the few elements allowed to occupy different surface positions will still be constrained to a limited area of freedom.

What kinds of constructions are liberating, compacting, or concatenating depends upon language-particular specifications. In languages that facilitate the identification of thematic relationships and constituency via a rich morphology (e.g. Warlpiri), a high number of liberating constructions will be called for, since fixed word order is not necessary to construct grammatical relationships. In contrast, a language like English will demand far fewer liberating constructions. Moreover, it is a matter of theoretical economy and empirical evidence as to whether a licensing construction or a linear precedence rule should explain a particular word order regularity. My intention, however, is not to decide such issues here; instead, I now turn to how the machinery described above can be used to capture the distribution of either with a minimal number of specifications.

\section{Linearization and either ... or constructions}

\subsection{Focus, compaction, and floating}

In this section, I build an analysis of either ... or constructions on the basis of a compacting construction for modifying disjunctions and an LP rule ordering either before contrastive focus. Working together with the assumptions of the symmetry condition and the left-edge hypothesis, these components explain many of the positions available to either and predict its absence in other linear positions.

The basic insight, building on den Dikken (2006), is that either must linearly precede any contrastive focus in the same word order domain. It is claimed here that either always attaches at the left edge of a flat $n$-ary branching disjunction, but the rule that licenses this structural combination also permits either to float around inside the disjunction. Limitations on the ordering of either stem from the linear ordering constraint that orders either before the contrastive focus. Further constraints emerge as the result of the word order domain compaction of other constituents, thus blocking any external domain objects from intervening between them. As we shall see in the next subsection, additional distributional constraints are attributable to limitations on ellipsis. 
To present this analysis formally, I begin by proposing that either is lexically specified as a disjunction modifier, i.e. it only modifies phrasal constituents marked as completed disjunctions (DISJ + ). Accordingly, the rule licensing the combination of either with other constituents reflects this (boxed indices indicate structure sharing of feature-values, e.g. in the example below, the MOD value of the left-hand daughter that specifies the modified expressions is required to be identical with the right-hand daughter):

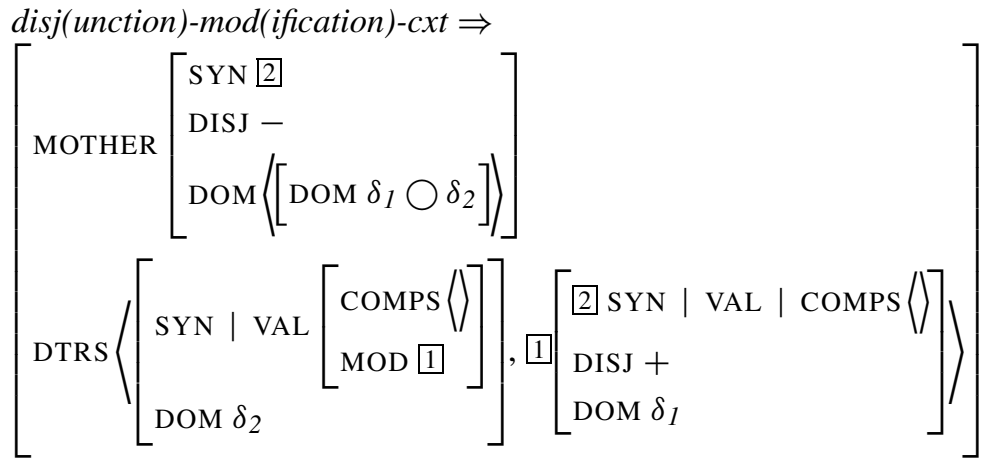

This rule licenses local trees where the right-hand daughter is a disjunction and the left-hand daughter modifies that disjunction (represented above via structure sharing). The syntactic mother inherits the DOM values of its daughters, shuffling them together onto a singleton DOM list. In other words, this is a type of compacting construction, following the constraints in (57). This means that once either combines with the disjunction, no other constituents can intermingle with the elements of the disjunction and vice versa. Such a constraint prevents either from ever "floating up"-it is trapped inside the disjunction by the compaction rule (upward floating, therefore, will be later characterized as an illusion). At the same time, however, the rule for compaction enables either to float around inside the disjunction, since the two lists $\delta_{1}$ and $\delta_{2}$ are shuffled together. In structural terms, either always attaches at the left edge of the disjunction because of this rule, in accordance with the left-edge hypothesis. While the mother inherits the syntactic type and values of the disjunction, it is no longer marked as a DISJ(UNCTION), which prevents stacking of either (*either either black or blue).

Disjunctions themselves are treated here as a type of coordination, consisting of a set of daughters $(1 \ldots \mathrm{k})$ along with the disjunctive coordinator (cf. Beavers and Sag 2004; Chaves and Sag 2010; Chaves 2008). As a subtype of coordination, disjunctions inherit all the constraints on coordination constructions in general (detailed subsequently). When the disjunction is complete, it is marked [DISJ +], allowing either to attach as desired.

By themselves, these specifications provide either with a high potential for linear freedom within a disjunction. In fact, without any other word order constraints, either could appear anywhere within a disjunction. Of course, a number of other constraints do play a role. As described above, some phrases (like NPs) compact when they are integrated with their lexical heads (Dowty 1995; Pollard et al. 1994; Chaves 2007). This will prevent either from intervening between the elements of NPs that have been 
integrated with their heads. Still, this does not explain the bad cases of (17)-(20) repeated here for reference:

(60) *John will read either CHAPTER 3 or prepare DINNER.

(61) *John will read either CHAPTER 3 or Jones will FLUNK him.

(62) JOHN $\langle *$ either $\rangle$ will $\langle *$ either $\rangle$ read $\langle *$ either $\rangle$ CHAPTER 3 or MARY CHAPTER 4.

The problem in each of these examples, as noted previously, is that either is within the interpretive scope of the contrastive focus.

Following other researchers (Engdahl and Vallduví 1996; De Kuthy 2002; De Kuthy and Meurers 2003; Maekawa 2004), I assume that signs contain informationstructure representations, expressed by the feature INFO(RMATION)-STRUCT(URE). This attribute bears the appropriate features FOCUS, CONTR(ASTIVE)-FOC(US), and TOPIC, which have lists of signs as their values, in the same manner as DOM values. Pitch accent values on lexical items act as the source of these focus values in English (Selkirk 1995; Engdahl and Vallduví 1996; De Kuthy and Meurers 2003). For instance, when the pitch accent is rising from low to high, the contrastive focus value is identified with the word itself. ${ }^{9}$ Other types of pitch accenting lead to different values for other information structure features (see above sources for further examples). When the accent on a word is unmarked, there is no contrastive focus contribution from that word.

For phrasal signs, i.e. non-terminal syntactic nodes, the contrastive focus values are instantiated according to focus projection principles. Contrastive stress on an object phrase in English can lead to the interpretation of narrow focus on just the object or the interpretation of wide focus on the verb phrase containing the object. These options are modeled in the HPSG accounts cited above by specifying that syntactic mothers can a) inherit or collect the focus values of the daughter nodes (i.e. narrow focus or focus inheritance) or b) receive a focus value identified with its own semantic contribution (i.e. wide focus or focus projection). ${ }^{10}$

In the situation where focus is interpreted widely, the focus value is identified with the syntactic mother, as depicted in the representation for the verb phrase in the left disjunct below:

\footnotetext{
${ }^{9}$ De Kuthy and Meurers (2003) argue that the type-identification is with the logical form or semantic contribution of the word, rather than the entire sign; however, this distinction does not directly affect the present analysis.

${ }^{10}$ Den Dikken opts for a $\theta$-path analysis after first considering a focus projection account where contrastive focus projects up to the node where either is placed. For him, one of the major problems with such an analysis concerns cases where either is left-displaced beyond the extent of contrastive focus. The present account does not run into such troubles, though, because contrastive focus does not play a role in determining where either attaches-it only sets a lower bound on floating. The other consideration that causes den Dikken to eschew focus projection is found in his assertion that Kiss (1998) shows that contrastive focus (or identificational focus, in Kiss' terms) does not project, in contrast to information focus. As noted by Büring (2006), though, Kiss does not actually make the case for two variants of intonationally marked focus, but rather that syntactic constraints interact with focus interpretation. Indeed, what Kiss labels "identificational focus" applies only to clefted NPs in English-thus, the extension of this notion to contrastive focus in other domains is not motivated.
} 
(63) He read CHAPTER 3 or prepared DINNER.

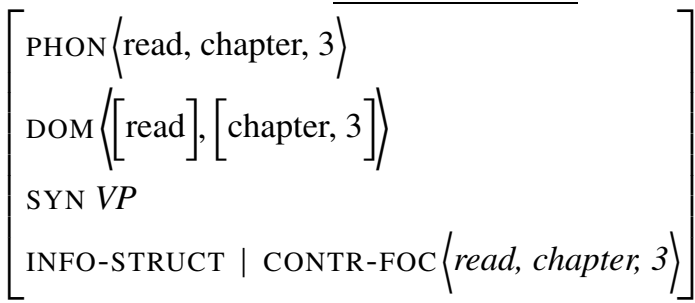

Even though intonational focus is only realized on the object NP in this example, the VP node reflects wide focus. For present purposes, therefore, the interpretive scope of the contrastive focus is identified with the CONTR-FOC value(s) of the highest syntactic node in a tree. ${ }^{11}$ Technically speaking, the domain elements themselves carry contrastive focus values, e.g. the contrastive focus value of the domain element corresponding to read in (63) is identified with its own semantic contribution since it is part of the wider contrastive focus.

In order to keep either away from the interior of the interpretive focus, I propose the following linear precedence rule that orders the disjunction-marking either before all contrastively focused material in the same word order domain $\left(\delta_{n e-l i s t}\right.$ stands for a non-empty list):

$$
\left[\begin{array}{l}
\text { PHON }(\text { either } \\
\text { SYN }[\operatorname{MOD}[\text { DISJ }+]]
\end{array}\right] \prec\left[\text { INFO-STRUCT }\left[\text { CONTR-FOC } \delta_{n e-l i s t}\right]\right]
$$

Such a linear precedence rule guarantees that either never surfaces after the contrastive focus in the left disjunct. Notice that such a linear precedence rule also ensures that either precedes or by at least one word, assuming that at least one word in every disjunct is contrastively focused (Hendriks 2003; Chaves 2007). While fo-

\footnotetext{
${ }^{11}$ The aforementioned focus accounts also present a number of constraints on focus projection. Engdahl and Vallduví (1996), for instance, assert that only the most oblique argument of a mother can project focus upward, while Selkirk (1995) states that a head or an internal argument can project focus. Büring (2006), however, presents compelling evidence that any sub-constituent can theoretically project focus to its syntactic mother, e.g.:
}

(1) a. Q: What will she do if her call doesn't go through again?

A: She'll [call him AGAIN $]_{\mathrm{FOC}}$.

[Adverb projection]

b. Q: Why did Helen buy bananas?

A: [Because JOHN bought bananas $]_{\text {FOC }}$.

[Transitive subject projection]

My aim here, though, is not to decide on the best theory of focus projection. From the specifications I have provided, it should be possible to determine for any disjunction sentence the set of possible contrastive focus assignments. Any focus theory, therefore, that successfully models the projection of focus from an accented constituent up to the interpretive scope of contrastive focus will be compatible with the account here. 
cused material often appears at the right-edge of the left disjunct, it is possible for it to appear earlier:

(65) *John gave ANGELA either the book or MARIE (the book).

Here, the linear precedence rule in (64) blocks either from appearing after the contrastively focused constituent in the same word order domain.

With these tools - the licensing construction for combining either with disjunctions and the linear precedence rule-the unlicensed positions for either in (60), depicted in (66), can be explained:

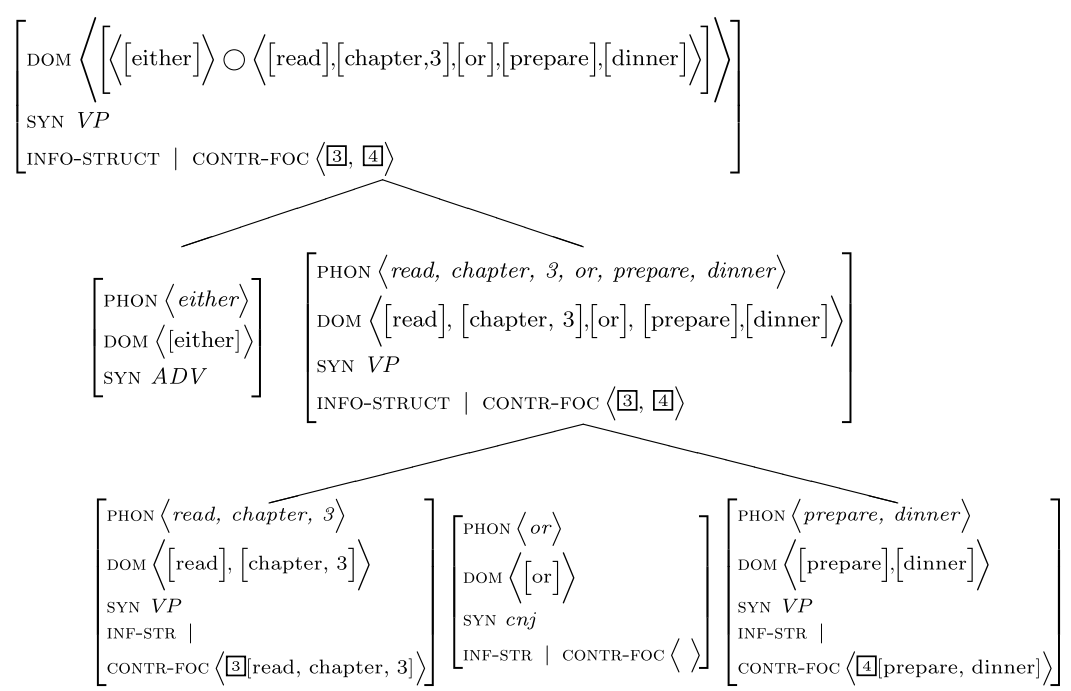

Here, the VP in the left disjunct is marked as having wide contrastive focus. This focused VP combines with the similarly focused right disjunct, yielding the coordinated VP with a focus contribution from each disjunct. At this point, either attaches, but it has nowhere to go: it cannot float inside the disjunction because the first element in the entire disjunction, read, is part of the contrastive focus. This leaves only one possible PHON value or word order possibility: 〈either, read, chapter, 3, or, prepare, dinner $\rangle$. This word order applies regardless of what precedes this disjunction, because the compaction principle that licenses the topmost node prevents either from entering into linear relations with any higher constituents.

Parallel accounts can be provided for (61) and (62), where either is banned from the scope of the contrastive focus. In these examples, either appears after at least one word in the contrastive focus, in conflict with the linear precedence rule. The constraint on disjunction modification predicts, though, that the modifier can surface quite far within the left disjunct, given that it comes before the contrastive focus. For instance, consider the example in (1), shown again in (67): 
(67) $S_{1}$ : Thomas will write one of two things.

$\mathrm{S}_{2}$ : 〈Either $\rangle$ Thomas $\langle$ either $\rangle$ will $\langle$ either $\rangle$ write $\langle$ either $\rangle$ A MYSTERY or he'll write A ROMANCE.

In this clause-level coordination, where either structurally attaches at the very beginning of the utterance, even positions past the verb are open to either. The disjunction modification and linear precedence rules predict just this:

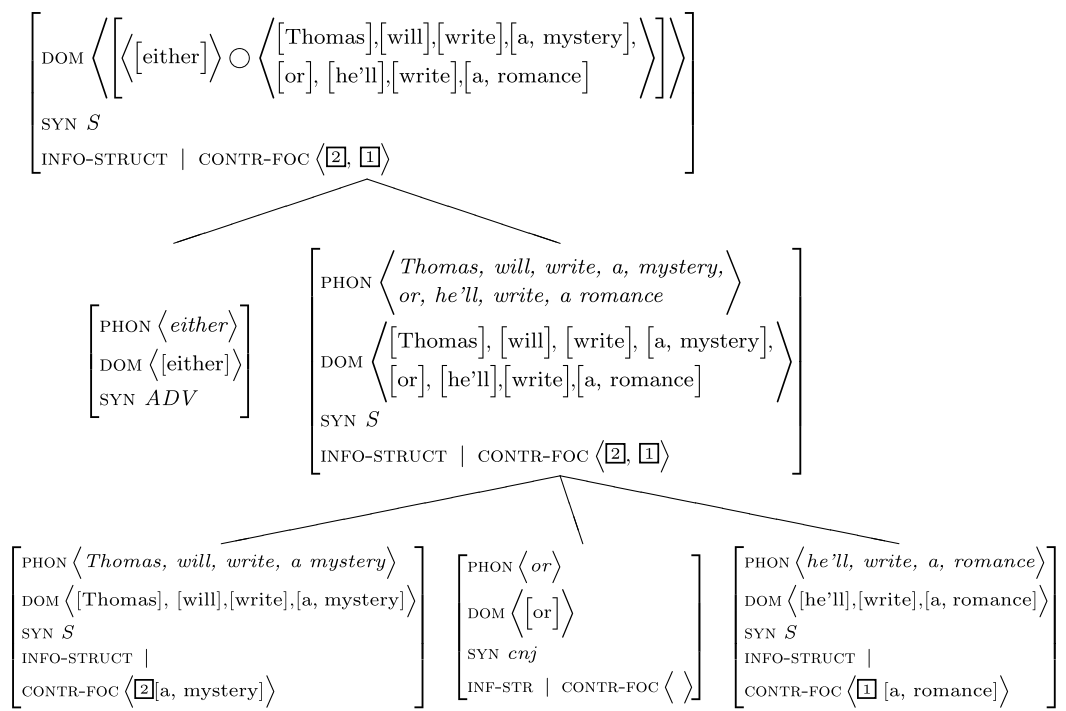

In each of the disjuncts, only the object NPs appear on the contrastive focus list (but other assignments are possible given a different discourse context). When either structurally attaches to the clausal disjunction, therefore, it can float down to the left edge of the contrastive focus. Since the subject, auxiliary, and verb are all nonfocused, either can appear before and after these domain elements. The consequence of this is four potential positions for either to land in and hence four possible PHON values.

Within this section, I have demonstrated how the rule for building either ... or constructions enables either to float into disjunctions. This constructional rule provides either with a great potential for linear freedom. At the same time, its positional variability is limited by (i) the extent of the interpretive focus and (ii) by the compaction process which keeps it inside the disjunction. Accordingly, while these rules allow for some linear freedom, they simultaneously impose strong boundaries on this freedom. As we shall see in the next section, further constraints on linear freedom emerge with the application of ellipsis.

\subsection{Coordinate ellipsis}

The above analysis captures the facts related to seeing either lower than expected, but does not explain the cases where either is "too high." Den Dikken tackles this 
problem by allowing base-generation of either at any point along the $\theta$-path. My analysis differs in that I suggest that either always attaches at the left edge of the disjunction, and in this section, following Schwarz (1999), I argue that ellipsis can account for the apparent unbalanced disjunctions where either is "too high."

Schwarz proposes that the surface form in (69a) has the underlying form shown in (69b), demonstrating a classic pattern of elision in coordinate constructions: shared material at the beginning of non-initial coordinates is deleted.

(69) a. Bernard either likes coffee or tea.

b. Bernard either [likes coffee or likes tea ].

On this analysis, the apparent coordination of different syntactic categories is illusory. Structurally speaking, either here attaches at the left disjunct edge, preserving the left-edge hypothesis. Similarly, the following examples that superficially result in unbalanced-looking disjunctions can be reanalyzed as symmetrical disjuncts:

(70) You'll either [need to bring a passport] or [need to bring a birth certificate].

(71) Either [Congress will pass the legislation] or [Congress will lose our confidence].

Den Dikken, too, acknowledges that at least some speakers accept either ... or constructions like (72) that must be analyzed with ellipsis of CP sub-constituents on his account, since the principles in his disjunctive generalization otherwise rule it out.

(72) He said either that he would eat RICE or that he'deat BEANS.

In addition to these precedents for an ellipsis account, there is also constituent-based and semantic evidence for ellipsis of shared left-peripheral material, as illustrated in the following examples:

(73) a. Simon either gave a cardigan to Vera or ave a jumpsuit to Ira.

b. Either Micah said that Will needed a rope or Micah said that Will needed a robe.

A jumpsuit to Ira does not constitute a well-formed constituent, which is reflected by the fact that it cannot be extracted (*It was a jumpsuit to Ira that Simon gave). An ellipsis-based account, however, can easily account for the grammaticality of sentences like (73a). Semantic evidence for the deletion process comes from examples like (73b), which has to be interpreted as saying that Micah said one of two things, rather than saying Will needed one of two things. Again, the correct reading emerges straightforwardly on an ellipsis account, while it is not clear how such a reading could be derived without ellipsis.

I accordingly adopt the idea that left-periphery ellipsis in disjunctions can account for surface positions of either that are higher than expected on the left-edge hypothesis. For the purpose of presenting an ellipsis-based analysis formally, I draw upon prior linearization research in HPSG on coordinate ellipsis (Beavers and Sag 2004; Chaves 2007; Chaves and Sag 2010). Within this research, left-periphery ellipsis in coordinations is licensed via the constructional rule that allows coordinations to be built. The interested reader may examine the formal representation of this rule in the 
Appendix section, but the constraints can be explicated without the technical apparatus, which is what I will do here.

The coordination construction rule expresses a simple generalization: shared material at the beginning of non-initial coordinates can be deleted, but unique material cannot be elided. Notably, this ellipsis-based rule is not intended as a comprehensive theory of ellipsis, as it only pertains to left-periphery ellipsis in coordinations. It does not, therefore, express constraints on deletion in other contexts or other kinds of deletion operations such as sluicing or VP ellipsis, or even other kinds of deletion processes in coordination, such as right-peripheral ellipsis (see Chaves 2007, 2008 for discussion). Despite this specificity, the licensing rule accounts for a wide array of coordination data, and it does so without introducing any new linearization machinery.

The basic idea is that we can arbitrarily split the linear sequence of words in a coordinate into two contiguous strings, call them A and B, the first of which can be empty, but the second cannot. Let us also assume that if $\mathrm{A}$ is non-empty, it contains a string beginning at the left edge of the coordinate. For instance, the coordinate likes coffee, can only be decomposed into the two following splits, given these constraints:

$$
\begin{aligned}
& \mathrm{A}=\langle\text { likes }\rangle, \mathrm{B}=\langle\text { coffee }\rangle \\
& \mathrm{A}=\langle\rangle, \mathrm{B}=\langle\text { likes, coffee }\rangle
\end{aligned}
$$

If we split all the coordinates of a coordination in such a way, we produce a series of A lists and B lists. Sometimes, the A lists will happen to match across coordinates. For instance, in the disjunction likes coffee or likes tea, the elements in the A lists will match when the verb from each disjunct is the single element on that coordinate's A list:

$$
\begin{aligned}
& \mathrm{A}_{1}=\langle\text { likes }\rangle, \mathrm{B}_{2}=\langle\text { coffee }\rangle \\
& \mathrm{A}_{2}=\langle\text { likes }\rangle, \mathrm{B}_{2}=\langle\text { tea }\rangle
\end{aligned}
$$

The coordination construction rule effectively says that, in such a situation, the A lists from non-initial coordinates are deleted when the coordinates are combined. Of course, deletion doesn't have to happen whenever coordinates are matched in terms of left-peripheral strings. This situation is modeled by allowing the A lists to be empty. In other words, deletion cannot target the elements in the B lists:

$$
\begin{aligned}
& A_{1}=\langle\rangle, B_{2}=\langle\text { likes, coffee }\rangle \\
& A_{2}=\langle\rangle, B_{2}=\langle\text { likes, tea }\rangle
\end{aligned}
$$

In the HPSG accounts cited above, the elements on these lists correspond to word order domain elements. Deletion occurs in these accounts when a word order element is not passed up in the tree. Hence, whenever the elements on the A lists match, the non-initial A lists will not be pronounced.

As an additional example, consider the disjunction in (73a), repeated in (77), where the VP disjuncts can be split into A and B lists as shown below:

(77) Simon either [gave a cardigan to Vera] or [gave a jumpsuit to Ira]

$\mathrm{A}_{1}=\langle[$ gave $]\rangle, \mathrm{A}_{2}=\langle[$ gave $]\rangle$,

$\mathrm{B}_{1}=\langle[\mathrm{a}$, cardigan], [to], [Vera $]\rangle, \mathrm{B}_{2}=\langle[\mathrm{a}$, jumpsuit $]$, [to], [Ira $\left.]\right\rangle$ 
Since the verb at the beginning of the first disjunct matches the verb at the beginning of the right disjunct, deletion of the latter verb is licensed. Identity matching is assessed on the basis of morphological lemma form and syntactic category information. ${ }^{12}$ Again, list divisions are non-deterministic, so a coordination can be associated with multiple, possible splits. In the case where all disjunct elements are pronounced, including shared material, then only the B lists are non-empty:

(78) Simon either [gave a cardigan to Vera] or [gave a jumpsuit to Ira].

$\mathrm{A}_{1}=\langle\rangle, \mathrm{A}_{2}=\langle\rangle$,

$\mathrm{B}_{1}=\left\langle[\right.$ gave], [a, cardigan], [to], [Vera] $\rangle, \mathrm{B}_{2}=\langle$ [gave], [a, jumpsuit], [to], [Ira] $\rangle$

The coordination construction rule consequently licenses coordinations with and without deletion of left-peripheral elements.

The final ingredient of this coordination construction rule is the specification that the antecedent of ellipsis compacts, meaning that once ellipsis has been triggered, no linear elements can intermingle with elements of the ellipsis antecedent. This feature of the coordination construction reflects the idea that ellipsis depends on matching the features of linear sequences, and that this identity match would be void if structurally higher elements were allowed to float into the linear sequence of the ellipsis antecedent, or if elements inside the antecedent were allowed to "float away." Without this compaction process, therefore, it would be ambiguous as to whether or not a floating element has a counterpart in the elided material.

The integrity of the matching process is thus ensured by the fact that the ellipsis antecedent is compacted in the coordinate's syntactic mother. The domain list of an ellipsis antecedent with four elements, $\langle a, b, c, d\rangle$, will accordingly be instantiated on the coordinate's mother as the singleton list $\langle[\langle a, b, c, d\rangle]\rangle$ (equivalently represented as $\langle[a, b, c, d]\rangle)$. Consider, for instance, the case where two identical VPs are coordinated with focus restricted to a post-verbal modifier, as in (79). The coordination construction licenses the optional deletion of material in the right disjunct.

Q: When should I do this?

A: [Do this TODAY] or [do this TOMORROW].

Given such ellipsis in the right disjunct, list $\mathrm{A}_{1}$ is non-empty and hence the domain elements in this set are compacted together on the disjunction's DOM list, i.e. $\langle[\mathrm{do}$, this $]\rangle$. What this means for either and any other floating elements is that they will be unable to intervene between elements of the ellipsis antecedent. Hence, only two positions for either are licensed-before and after the ellipsis antecedent-while the position between verb and object is unavailable.

(80) $\langle$ Either $\rangle$ do $\langle *$ either $\rangle$ this $\langle$ either $\rangle$ today or do this tomorrow.

Similarly, this compaction process makes sense of the contrast between the (a) and (b) examples below, originally presented by den Dikken:

\footnotetext{
${ }^{12}$ Establishing identity on the basis of morphological lemma form, rather than phonology, allows deletion in spite of agreement mismatches like There were two guards when I arrived and one when I left. See Chaves and Sag (2010) for a related alternative, where semantic restrictions govern left-periphery ellipsis.
} 
(81) a. John put $\langle *$ either $\rangle$ the book 〈either $\rangle$ on the SHELF or on the TABLE.

b. John put $\langle$ either $\rangle$ the BOOK $\langle *$ either $\rangle$ on the SHELF or the RECORD on the TABLE.

In (81a), the licit position of either immediately before the PP is licensed by ellipsis of the verb or the verb and direct object, e.g. put the book on the shelf or put the book on the table. No matter what the scope of elision is, either will be safely positioned after it. The position before the nominal object, however, is predicted to be unavailable. A parse with elision of only the nominal object in the second disjunct rules this position out because it would entail the coordination of non-constituents $\left([\text { the book }]_{N P}[\text { on the shelf }]_{P P}\right.$ or $\left.[\text { the book }]_{N P}[\text { on the table }]_{P P}\right)$. A parse with elision of any more material (e.g. put the book) would block either from interrupting the compacted ellipsis antecedent-thus leaving no possible grammatical parse. To see this, examine the representation where either has combined with a disjunction where ellipsis has applied to the right-disjunct string, put the book:

$$
\left[\begin{array}{l}
\operatorname{DOM}\langle\text { either }\rangle \bigcirc\langle[\text { put, the, book }],[\text { on }],[\text { the, shelf }],[\text { or }],[\text { on }],[\text { the, table }]\rangle \\
\text { SYN VP } \\
\text { INFO-STRUCT I CONTR-FOC }\langle[\text { the, shelf }],[\text { the, table }]\rangle
\end{array}\right]
$$

Although either can float in the left disjunct, it can only occupy positions between domain elements. Since the ellipsis antecedent has now been effectively transformed into a single domain element, either cannot appear between its elements. For (81b), the illegal position is clearly ruled out because either fails to precede the contrastively focused theme. The acceptable position is predicted, too, given deletion of the verb (or subject and verb) in the second disjunct. In either case, either is outside the scope of the ellipsis antecedent. The domain compaction of ellipsis antecedents thus further greatly reduces the linear possibilities open to either in the context of elision, effectively limiting it to a position immediately before or immediately after the ellipsis antecedent.

One of the central merits of the coordinate ellipsis analysis in general is that it accounts for a range of coordination phenomena that are otherwise problematic, including the seeming coordination of unlikes (Beavers and Sag 2004):

(83) Jan [is a Republican and is proud of it].

(84) Sam [gave a car to his daughter and a motorcycle to his son].

At the same time, ellipsis is not unconstrained in this theory. The requirements for morphological lemma form and category matching exclude the critical data discussed in Wilder (1997) on content-identity, (85a), while the constraint that only shared material in non-initial coordinates can be deleted accounts for the strict directionality of these deletion processes, (85b):
a. *[John came in] and [Mary sat down].
b. *[Mary came in] and [Mary sat down]. 
Furthermore, the coordination construction rule only permits deletion of shared left edge material. Because of this, it will not license ellipsis of shared phrases if the identity-match does not extend to the left edge of each disjunct: ${ }^{13}$

*The book pleased John and John bought it.

Overall, this ellipsis mechanism is quite powerful, as it does not itself place constraints on the kinds of syntactic elements that can be omitted or the kinds of elements that can be left behind. It is, however, constrained to apply to only word order domain objects and cannot target phonological material inside a single domain object. In other words, it cannot split apart linear elements that have been compacted, because these elements constitute a single domain object. For example, since NPs that have been integrated with their heads are systematically compacted, the coordination construction does not allow these NPs to be split apart. Thus, the left periphery of an argument NP cannot be deleted:

(87) *The best swimmer lost and the best runner won.

*The boy gave a book to Mary and ave a rose to Sue.

*John revised either his decision to cook rice or his decision to cook beans.

The prohibition on deleting part of a compacted domain also pinpoints the problem with either ... or constructions like (89), where part of the complex NP must be deleted. A minimally different version of (89) without either is perfectly acceptable, however, because it can be parsed without any ellipsis in the second disjunct. Thus, while the coordinate ellipsis mechanism itself is insensitive to the syntactic properties of the elided material (as long as category identity holds), other aspects of the grammar interact with this mechanism to limit the available targets for deletion.

More generally, this ellipsis mechanism contrasts with other approaches to leftperipheral deletion that assume the base-generation of empty elements (Wilder 1997) or deletion of syntactic structure (Wexler and Culicover 1980; van Oirsouw 1987). In the current model, syntactic structure is unaffected by the ellipsis operation. Ellipsis operates here at the level of word order domains, which are subject to constraints that are independent of the constraints on hierarchical structure. This strategy has several desirable consequences. First of all, the ellipsis process can target constituents and non-constituents alike:

(90) I [sent a postcard to your brother on Monday and sent a pesteard to your sister on Tuesday].

The most salient interpretation for this example is that different postcards were sent, which accords with the deletion-based analysis. Moreover, as evidenced by earlier examples, the remnants of ellipsis need not be constituents either. These facts are surprising if deletion operates at the level of syntactic structure, but wholly unremarkable

\footnotetext{
${ }^{13}$ Note that this requirement will also guarantee the satisfaction of Wilder's (1997) Head Condition on Forward Deletion in coordinations: "An ellipsis site may not be c-commanded by an overt (non-deleted) head in its domain (=conjunct)" (p. 74). Some adverbial remnants, however, are permitted before the ellipsis site, as in Sandy will offer a record to Chris and not a book to Lee (Chaves 2007: 341).
} 
if ellipsis operates at the level of linear order. Additionally, the linearization-based account of left-peripheral ellipsis can account for the deletion of word-parts, as in the following example (Chaves 2008):

(91) House-cleaning and house-repairing is an ongoing process for many people.

If lexical compounds can consist of multiple domain objects, then the coordination construction rule will straightforwardly allow omission of the left-peripheral noun in the right coordinate. The possibility of omission of word-parts according to similar principles as linear strings emerges straightforwardly from the linearization account, but is not transparently derivable from syntactic deletion accounts.

The base-generation accounts, too, encounter some difficulty with respect to their underlying assumptions. For instance, the assumption in Wilder (1997) that empty elements can project syntactic structure conflicts with Minimalist assumptions about syntactic projection. Consequently, Wilder assumes that there are special lexical variants of ordinary lexical items, except that they lack phonological content. Besides the theoretical problems that such an assumption produces (e.g. how to control for a proliferation of phonologically empty elements), the ellipsis constraints are difficult to evaluate due to a lack of precision. For instance, the Context Identity constraint requires that an ellipsis antecedent and the ellipsis target have the same structural role within their coordinates. As Chaves (2008) points out, not only is such a constraint computationally non-trivial, but there is no explicit account of how such a constraint could be formulated in the Minimalist framework.

The linearization-based ellipsis account described here thus can account for the apparent insensitivity of ellipsis to syntactic constituency, the parallels in word-part ellipsis, as well as the conundrum presented by the coordination of unlikes. In addition, the coordination construction rule defines a set of precise, well-defined constraints that formally characterize the conditions under which left-peripheral ellipsis in coordinations can apply. These constraints can be summarized as follows:

(i) Only left-peripheral strings in non-initial coordinates can be elided.

(ii) Elision of left-peripheral strings requires matching morphological lemma and syntactic category information for each domain element in the scope of ellipsis.

(iii) Each coordinate must pass up some non-empty, word order domain list.

(iv) The antecedent of ellipsis is compacted on the coordinate's syntactic mother.

Due to these considerations, and the fact that the linearization account provides a ready-made way to model the interactions between morphological, phonological, semantic, and syntactic constraints, the present left-peripheral ellipsis account provides an excellent means for modeling ellipsis in either ... or constructions.

\subsection{Data overview}

Having now assembled all the necessary tools for modeling the word order freedom of either in disjunctions, we can see how it works in action. The coordination construction and disjunction modification rules now can be used to visualize the steps by which either appears higher than expected, as seen in (92): 


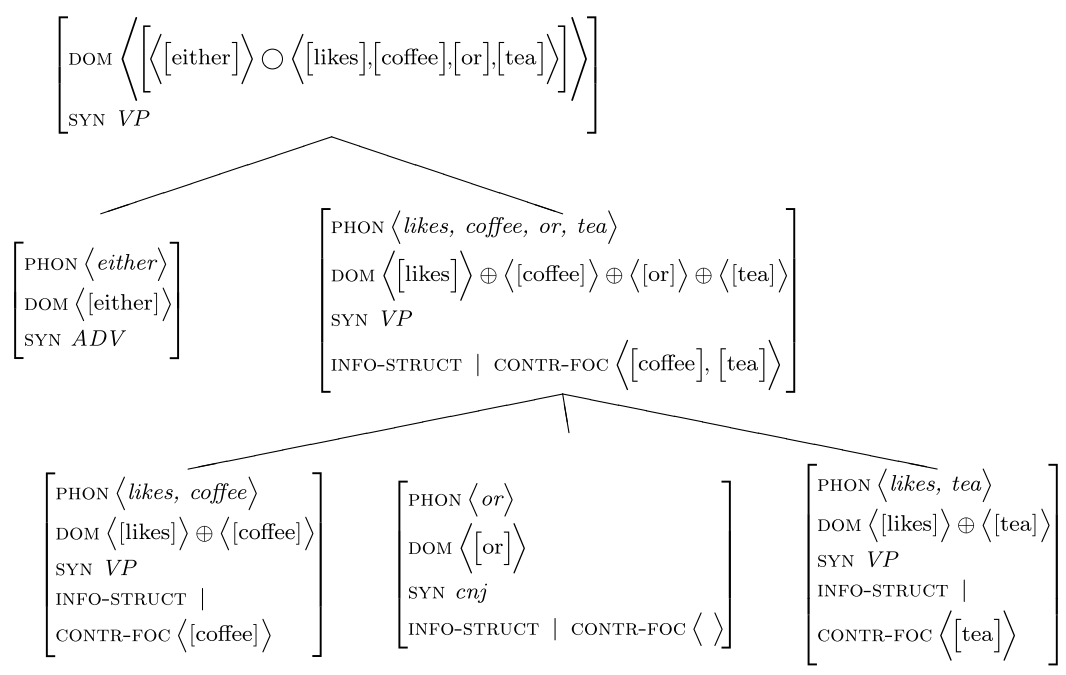

The coordination is instantiated according to the principles for coordination constructions, meaning that the right-hand verb which shares its form and head values with the verb in the left disjunct can be deleted. The ellipsis antecedent compacts once this process is triggered, although the fact that the antecedent is only one word makes the process rather moot in this case. Only a single word in each disjunct (coffee and tea) is contrastively focused here (although alternative focus assignments are possible), so either is not restricted from positions prior to these words. The result is that either actually has two possible surface positions: before and after the verb. When it occurs prior to the verb, this creates the superficial effect of unbalanced disjunctions.

Examine, as well, the original data presented at the beginning of this article. The unlicensed positions in (8)-(11), repeated as (93)-(96), violate one of the word order constraints posited here.

(93) Thomas will read $\langle *$ either $\rangle$ a book or take a nap.

(94) Thomas will read a 〈*either $\rangle$ book or take a nap.

(95) Thomas will read a book $\langle *$ either $\rangle$ or take a nap.

(96) Thomas gave Angela 〈*either〉 the book or Marie (the book).

(93) involves either inside the scope of contrastive focus; (94) has either inside a compacted NP; in (95) and (96), either again appears after the contrastive focus. In contrast, the legitimate positions are licensed via downward floating and/or ellipsis. For instance, in (2)-(4) [(97)-(99)], the "too high" positions reflect elision in the right disjunct. The amount of elided material depends upon the position of either:

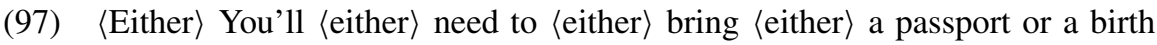
certificate. 
(98) 〈Either $\rangle$ Congress $\langle$ either $\rangle$ will $\langle$ either $\rangle$ pass the legislation or lose our confidence.

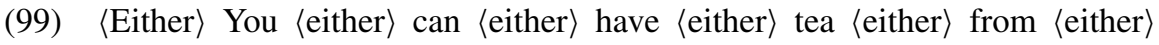
China or from Tibet.

The higher either shows up in these superficially unbalanced-looking disjunctions, the more material must be elided. Of course, when either sits at the edge of a balanced-looking disjunction, no elision is necessary. In (97), either can surface past the main verb, since the verb bring is not focused. In (98), though, either cannot appear to the right of the verb, pass, which is part of the contrastive focus. (99) presents a more extreme case, where either can float into a prepositional phrase, as the only contrasted material is the prepositional object.

The linearization-based constraints further explain why either appears insensitive to contrastive focus at times. Recall that the idea that either must c-command contrastive foci was ultimately rejected by den Dikken, based partly on multiple foci examples like (24)[100], where either is restricted from a position above the hierarchically higher focus:

(100) Q: Did John say that he had either FRIED it or BAKED it?

A: No! John 〈*either〉 DENIED 〈*either〉 that 〈*either〉 he 〈*either〉 had $\langle$ either $\rangle$ fried it or baked it.

This fact falls out naturally on the present account without requiring the abandonment of the linear precedence rule. The reason for this is that linear precedence rules operate only on DOM elements that belong to the same list (i.e. elements in the same linear order domain), and once either attaches to a disjunction, the disjunction modification construction closes off that list. Consider the following question-answer pair to illustrate this scenario:

(101) Q: Will you order either THE MOUSSE or THE SOUFFLÉ?

A: Neither, but $\langle *$ either $\rangle$ ADAM will 〈either〉 order 〈either〉 THE MOUSSE or THE SOUFFLÉ.

Here, either can attach to the coordination of order the mousse or erder the soufflé. The disjunction modification construction licenses this combination and the following feature-value specifications:

$$
\left[\begin{array}{l}
\operatorname{DOM}\langle[\operatorname{DOM}\langle[\text { either }]\rangle \bigcirc\langle[\text { order }],[\text { the, mousse }],[\text { or }],[\text { the, soufflé }]\rangle]\rangle] \\
\text { INYN VP }
\end{array}\right.
$$

Only a single domain element appears on the domain list of this coordinated VP. Hence, either is only sensitive to linear precedence rules inside of this compacted domain. In this case, the linear position of either is again restricted to before or after the verb. Combining this coordinated VP with the higher syntactic constituents cannot 
change these linear order facts, due to the persistence constraint. When the contrastive subject combines with the rest of the clause, either remains inside the compacted coordination, as shown in the following S-level representation where either has been (arbitrarily) positioned before the verb.

$$
\left[\begin{array}{l}
\operatorname{DOM}\langle[\text { Adam }],[\text { will }],[\operatorname{DOM}\langle[\text { either }],[\text { order }],[\text { the, mousse }],[\text { or }],[\text { the, soufflé }]\rangle)\rangle \\
\text { SYN S } \\
\text { INFO-STRUCT I CONTR-FOC }\langle[\text { Adam }],[\text { the, mousse }],[\text { the soufflé }])
\end{array}\right.
$$

In essence, therefore, either is oblivious to the fact that the subject is also contrastively focused because they are in different word order domains. Because word order domains restrict the application of linear precedence rules and the possibility of domain merging, examples like these do not counterexemplify the proposed constraint that either must precede contrastive focus.

As to why either cannot be positioned before the contrastive subject, the answer is that the subsequent string does not constitute a well-formed disjunction. Without focus on the subject, such a string could be a legitimate disjunction, but only given ellipsis in the right disjunct; however, focused constituents cannot be deleted (Heim 1997; Romero and Han 2003): ${ }^{14}$

\section{*ADAM will order THE MOUSSE or ADAM will order THE SOUFFLÉ.}

Furthermore, the validity of the claim that either can only attach to the superficial left edge of the disjunction in examples like (24) and (101) is largely contingent on the position of either in the soliciting question. Thus, if the question was reformulated as follows, a parallel position for either sounds acceptable:

Q: Did John say that he either had FRIED it or BAKED it?

A: No! John DENIED that he either had fried it or baked it.

Similar question-answer pairs can be constructed for the other positions below the matrix verb. Admittedly, the sentences are increasingly degraded the farther either is dislocated from the edge of the disjunct-internal contrastive focus, but this correlation holds generally in either... or constructions, so no special significance should be attributed to it here. Thus, the concern that either seems insensitive to certain contrastive foci is expected on the linearization account: domain compaction effectively limits the attention of either to the focus in the disjunction. Contrastive foci outside that linear order domain are immaterial to the position of either.

The examples presented to forge an analysis of $o r$-movement also have alternative explanations on the ellipsis approach. For instance, it is observed that either cannot

\footnotetext{
${ }^{14} \mathrm{An}$ anonymous reviewer raises the question of whether the second instantiation of the subject must be focused, i.e. whether the underlying structure for examples like (72) could not be, ADAM will order THE MOUSSE or he'll order THE SOUFFLÉ. In such a case, though, the coordination construction rule would not allow deletion to proceed due to the mismatch between FORM values of the two subjects. Moreover, since the relevant material has been deleted, it would be effectively impossible to support the hypothesis that the underlying form has changed.
} 
appear underneath negation in clause-level disjunctions, like (106), nor above negation in examples like (107):

(106) *Micah doesn't either SMOKE or he doesn't DRINK.

*Micah either doesn't SMOKE or DRINK.

Den Dikken's analysis of (107) depends upon the assumption that "negation breaks the $\theta$-path leading up from the contrastive focus" (p. 718). But such an analysis does not account for why either is unacceptable in clausal coordinations like (106), because either is allowed to attach directly to the first constrastive focus. This accordingly motivates an explanation in terms of conditions on the movement of or. In particular, it is suggested that negation creates a type of island boundary that or cannot cross, as discussed earlier.

On the present analysis, examples like (107) are excluded if negation is simply not elidable. Since either never floats leftward on the linearization account, its position in front of negation can only lead to two parses of the disjunction: (i) two negated VPs or Ss are being contrasted with elision of the negation in the second disjunct or (ii) the second disjunct does not underlyingly contain negation, in which case there is a number agreement error on the second verb. That there are clearly constraints on the deletion of negation is evident from examples like the following observations that originate with Ross (1967):

*Kim doesn't like Sandy and Lee deesn't like Pat.

*Kim will not read that book and Sandy will not either.

In fact, den Dikken explicitly identifies the deletion of negation as the source of unacceptability for IP-level parses of the disjunction in examples like (107). Unlike the present account, however, den Dikken's analysis does not require a parse with ellipsis (to avoid an agreement error), and consequently must invoke other factors to capture the unacceptability of such disjunctive structures.

In turn, the deviance of (106) can be argued to stem from the fact that either is inside the interpretive scope of the contrastive focus: the negation is part of the interpretive contrast. This accords with the strong intuition that not smoking and not drinking are being contrasted in the example below, rather than the actual habits of smoking and drinking: ${ }^{15}$

(110) Micah either doesn't smoke or he doesn't drink.

(110) is a natural follow-up to the statement that Micah has one of two characteristics. Interpreting clausal negation as part of the interpretive focus thus resolves the question of why either cannot appear past it in the surface string: such a word order violates the linear precedence constraint ordering either before contrastive focus.

\footnotetext{
${ }^{15}$ This leads to the question of why negation must always be part of the interpretive scope of contrastive focus in clause-level disjunctions. Contrasting two parts of an event or a proposition as in Hugh likes either cakes or Hugh likes pies compares similar predications that only differ with respect to the type of object. But two individually negated VPs do not overlap at all in event type, and so must be completely contrasted.
} 
Den Dikken's account, of course, could theoretically be supplemented with a similar mechanism for coordinate ellipsis. Indeed, nothing in that account rules out the possibility that ellipsis may explain some of the distributional data. Hence, some of the limitations of his disjunctive generalization in (25) could be theoretically circumvented by allowing ellipsis in exceptionally high surface positions of either. As noted in Sect. 3, the analysis embodied in (25) does not predict the possibility of examples like (111) without ellipsis, since CP blocks the $\theta$-path projected from the adverb:

(111) Depending on those values, the equation may either [suggest that life arises frequently] or [stggest that life arises infrequently].

If left-peripheral ellipsis of non-constituents is permitted, however, such examples could be described as either attaching to the left edge of the VP disjunction. In general, "high" positions of either could be interpreted as base-generation of either at the edge of the disjunction, while disjunction-internal deletion processes account for the superficial imbalance.

There are, however, remaining empirical coverage issues that separate the present account from that of the earlier focus-based approach, even after supplementing the latter with ellipsis. In particular, or-movement is postulated to explain the ungrammaticality of negation examples like (106), but also certain complex NP examples:

(112) *John was reading a book either about Chomsky or he was reading a book about chopsticks.

On the view that or is a phrasal category that moves from a position parallel to $e i$ ther to its surface position, or should generally not be able to escape islands. The evidence, however, reveals numerous examples that would necessitate or shifting out of established island environments:

(113) a. ... consider whether you should either cancel these projects, or whether you should raise their priority.

[www.mindtools.com/pages/article/newHTE_83.htm]

b. ... this will determine whether you are here either to honestly find the truth or whether you are here to just defend an intellectual position.

[galatiansc4v16.wordpress.com/2006/08/31/the-emergent-cult-listen-to-pastor-ken-silvas-warning-to-the-church]

c. One answer is to stop the search process when you either get bored or when you have what you need.

[advogato.org/article/142.html]

Such word orderings are predicted to be possible on the linearization analysis. For instance, (113a) is compatible with a CP-level disjunction parse with either attaching at the left edge. Since this example supports a focus interpretation where focus does not extend to the auxiliary (e.g. You should consider whether to do one of two things), either can intermingle with the $\mathrm{CP}$-internal constituents and surface before or immediately after the modal auxiliary. In contrast, disjunctions like (112) with either inside the complex NP are ruled out by the constraint that says that argument NPs compact when they are saturated by their syntactic heads. Thus, the IP-level disjunction in (112) will have the domain specification below, effectively blocking either from entering the complex NP. 


$$
\left[\begin{array}{l}
\operatorname{DoM}\left(\begin{array}{l}
{[\text { John }],[\text { was }],[\text { reading }],[\text { a, book, about, Chomsky }],[\text { or }],} \\
{[\text { John }],[\text { was }],[\text { reading }],[\text { a, book, about, chopsticks }]}
\end{array}\right]
\end{array}\right.
$$

There is thus no appeal to islands per se on the present account, but rather the observation that certain word order domains compact in systematic ways.

Beyond these issues, the previous focus-based analysis requires the already discussed ad hoc stipulation that either is sometimes insensitive to focus and must instead be placed on the first disjunct. This stipulation accounts for multiple foci examples like (101), IP-level disjunctions with negation, and CP-coordinations with either outside the $\theta$-path (111). Since focus plays such a critical role for certain examples, it is unclear why its importance is demoted in others. The absence of such an explanation prevents us from being able to predict whether focus will matter or not for any given example. It is only ex post facto that we can say if focus matters, thereby applying a descriptive label without understanding why it applies. In contrast, the linearization account here maintains a constant relationship between the scope of the interpretive focus and the word order possibilities for either.

The present linearization approach raises the question, however, of the possible extent of ellipsis and thus "how high" either can appear. On the present account, nothing limits the quantity of words or phrases that can be deleted from the left periphery of a disjunction, given the proper structure sharing. Nevertheless, constructed examples with either placed extremely high do sound degraded, especially when clause boundaries are included in the deleted material. The absence of grammatical constraints on the size of ellipsis, however, does not preclude the existence of performance-based preferences. Martin and McElree (2008), for instance, report that additional NP arguments in the antecedents to verb phrase ellipsis decrease performance accuracy in speed-accuracy tradeoff tasks. That is, comprehension-based performance decreases in (115b), which contains an additional NP (the painter), compared to (115a):

a. The photographer questioned who to hire, but the gallery owner realized that the famous art critic did not.

b. The photographer questioned who the painter should hire, but the gallery owner realized that the famous art critic did not.

It is thus not unreasonable to suppose that complexity-based effects at the discourse or referential level may influence acceptability judgments of either ... or constructions. In addition to the complexity of the deleted material, there may also be secondary processing-driven constraints, such as a preference for either to be close to the focus.

In sum, in this linearization-based account of either ... or constructions, either always attaches at the left edge of the disjunction in accordance with the left-edge hypothesis. When either looks to be dislocated to the left, this is actually an illusion caused by elision. Dislocation inside the disjunction, however, results from the hypothesized dissociation between hierarchical structure and linear order. Either can float into the left disjunct provided the entire disjunct is not contrastively focused. Although both this analysis and den Dikken's guarantee that either comes before the contrastive focus, they do so in substantially different ways. Here, this guarantee stems from the linear precedence rule. In den Dikken's account, this results from 
constraints on the base-generation of either, along with movement constraints on or. On the linearization account, therefore, the "too high" and "too low" problems actually do involve two different explanations: elision and word order shuffling. The utilization of these tools ultimately leads to a resolution of the problem posed by $e i$ ther. Neither the left-edge hypothesis nor the symmetry condition on coordination is counter-evidenced by either ... or constructions. Both principles can be maintained on the view that there is potential dissociation between hierarchical structure and linear order.

\section{Conclusion}

The focus-based analysis of den Dikken (2006) was revised here by capitalizing upon the capabilities of linearization tools. The insight that either cannot follow the contrastive focus was key to this analysis. The remainder of the distributional facts related to either ... or constructions were derived from a small set of assumptions: either combines with disjunctions via a compacting constructional rule and some syntactic constituents, such as integrated NPs, regularly compact in English. The compacting constructional rule provides either with linear freedom inside the disjunction due to the domain merging, while simultaneously keeping it away from unrelated foci.

The arguments and linearization machinery presented here accomplish more than simply predicting the available linear spots open to either. The accumulated evidence suggests that linearization theory has an important role in the syntactic analysis of even relatively fixed word order languages. Little has been said in the syntactic literature about word order variability in English for understandable reasons; however, the conclusion from the analysis developed here should be that understanding such word order variability can actually simplify syntactic analyses overall. While the linearization tools covered here may show some formal complexity, they have a wide range of application and express some relatively simple intuitions about word order. Compaction rules, for instance, merely state that certain strings may not enter into linear precedence relations with other strings, although the underlying reasons for this may vary from construction to construction. Greater attention to linear precedence rules and linearization constraints may thus provide a means to further streamline the grammars that linguists hypothesize, even in languages and constructions that exhibit relatively fixed word order.

Acknowledgements For valuable comments on this manuscript, I thank Peter Sells, Ivan Sag, and Rui Chaves, and the three anonymous reviewers. The editor, John Moore, deserves special thanks for carefully guiding the revisions of this manuscript. All errors and inaccuracies are my own.

Open Access This article is distributed under the terms of the Creative Commons Attribution Noncommercial License which permits any noncommercial use, distribution, and reproduction in any medium, provided the original author(s) and source are credited.

\section{Appendix}

The formal description of the coordination construction rule is given below: 


$$
\begin{aligned}
& \text { coord-cxt } \Rightarrow \\
& \text { for } n \geq 0, k \geq 2
\end{aligned}
$$

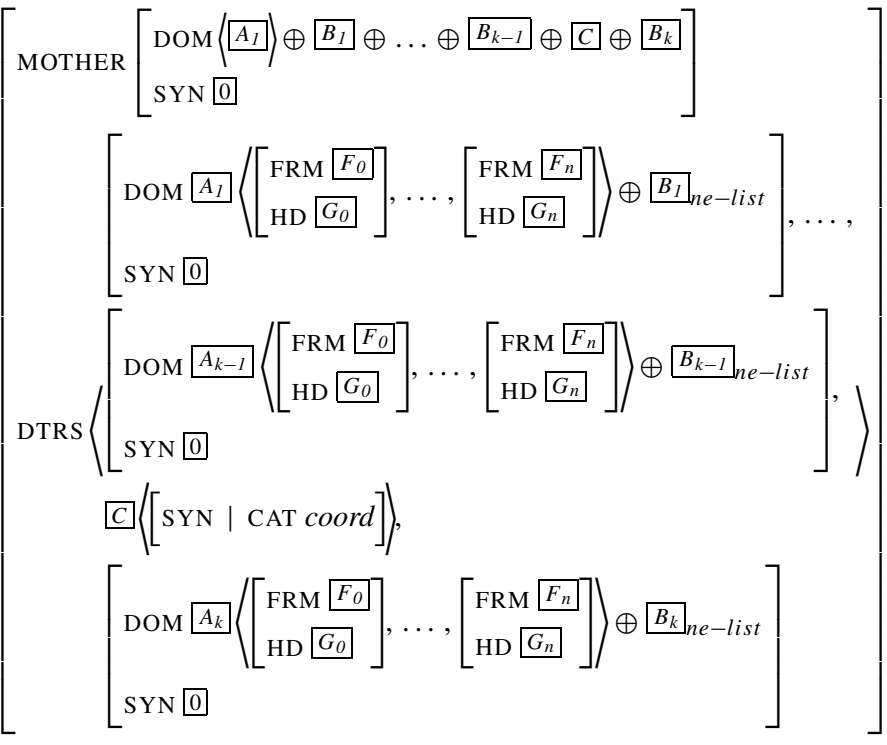

According to (116), the word order domain of a coordination can contain a subset of the phonological constituents belonging to the individual coordinates (which are constrained to have identical syntactic attributes). This optionality is captured by the fact that all of the DOM elements on the daughters' lists do not appear on the mother's DOM list. Each daughter's word order domain list is split non-deterministically into two lists, the first of which can be an empty list $(n=0)$, while the second cannot. Any unique material in the coordinates (here $B_{l} \ldots B_{k}$ ) always gets included in the mother's domain; thus, unique material is always pronounced. $B_{l} \ldots B_{k}$, however, can contain shared material as well, to account for coordinations with shared, overt strings, e.g. Sam skis well or Sam kayaks well, where $B_{1}=$ Sam skis well, $B_{2}=$ Sam kayaks well,,$C=$ or and all other lists are empty.

Deletion of shared left-peripheral material requires the non-emptiness of the domain lists $A_{l} \ldots A_{k}$, which are constrained to have elements with the same morphological lemma form and category. When these lists are resolved as non-empty, then only the corresponding signs from the initial coordinate are passed up to the mother's DOM value. Because of the correspondence between the PHON value of a sign and the values on the DOM list, the shared material in the non-initial coordinates can be left unpronounced. If $A_{I}$ is non-empty, i.e. ellipsis has occurred, then the ellipsis antecedent is compacted on the mother's domain, as reflected by the fact that $A_{l}$ is itself inside a list on the mother's DOM specification.

\section{References}

Beavers, John, and Ivan A. Sag. 2004. Coordinate ellipsis and apparent non-constituent coordination. In Proceedings of the HPSG04 conference, ed. Stefan Müller. Stanford: CSLI.

Büring, Daniel. 2006. Focus projection and default prominence. In The architecture of focus, eds. Valéria Molnár and Susanne Winkler. New York: Mouton de Gruyter. 
Chaves, Rui P. 2007. Coordinate structures—constraint-based syntax-semantics processing. PhD Thesis, University of Lisbon.

Chaves, Rui P. 2008. Linearization-based word-part ellipsis. Linguistics and Philosophy 31: 261-307.

Chaves, Rui P., and Ivan A. Sag. 2010. Left- and right-peripheral ellipsis in coordinate and non-coordinate structures (submitted).

Chomsky, Noam. 1957. Syntactic structures. The Hague: Mouton de Gruyter.

Chomsky, Noam. 1973. Conditions on transformations. In A Festschrift for Morris Halle, eds. Stephen Anderson and Paul Kiparsky, 232-286. New York: Holt, Reinhart \& Winston.

Chomsky, Noam. 1986. Barriers. Cambridge: MIT Press.

De Kuthy, Kordula. 2002. Discontinuous NPs in German-a case study of the interaction of syntax, semantics and pragmatics, Stanford: CSLI.

De Kuthy, Kordula, and W. Detmar Meurers. 2003. The secret life of focus exponents, and what it tells us about fronted verbal projections. In Proceedings of the HPSG03 conference, ed. Stefan Müller. Stanford: CSLI.

den Dikken, Marcel. 2006. Either-float and the syntax of co-or-dination. Natural Language \& Linguistic Theory 24: 689-749.

Donohue, Catherine, and Ivan A. Sag. 2004. Domains in Warlpiri. Unpublished manuscript, Stanford University.

Dowty, David. 1995. Toward a minimalist theory of syntactic structure. In Discontinuous constituency, eds. H. Bunt and A. van Horck, 11-62. Berlin: Mouton.

Engdahl, Elisabeth, and Enric Vallduví. 1996. Information packaging in HPSG. In Edinburgh working papers in cognitive science, eds. Claire Grover and Enric Vallduví, Vol. 12 of Studies in HPSG, 1-31. Edinburgh: Center for Cognitive Science, University of Edinburgh.

Ernst, Thomas. 2002. Syntax of adjuncts. Port Chester: Cambridge University Press.

Ernst, Thomas. 2004. Principles of adverbial distribution in the lower clause. Lingua 114: 755-777.

Gazdar, Gerald, and Geoff Pullum. 1981. Subcategorization, constituent order, and the notion 'head.' In The scope of lexical rules, eds. Michael Moortgats, Harry van der Hulsts, and Teun Hoekstras. Dordrecht: Foris.

Han, Chung-hye, and Maribel Romero. 2004. The syntax of whether/Q ... or questions: ellipsis combined with movement. Natural Language \& Linguistic Theory 22: 527-564.

Heim, Irene. 1997. Predicates or formulas? Evidence from ellipsis. Proceedings of SALT 7: 197-221.

Hendriks, Petra. 2001. Initial coordination and the law of coordination of likes. In Linguistics in the Netherlands 2001, eds. Ton van der Wouden and Hans Broekhuis, 127-138. Amsterdam: John Benjamins.

Hendriks, Petra. 2003. Either is a focus particle. Unpublished manuscript, University of Groningen.

Hendriks, Petra. 2004. Either, both and neither in coordinate structures. In The composition of meaning, eds. Alice ter Meulen and Werner Abraham. Amsterdam: John Benjamins.

Kathol, Andreas. 2000. Linear syntax. Oxford: Oxford University Press.

Kathol, Andreas, and Carl Pollard. 1995. Extraposition via complex domain formation. In Proceedings of the thirty-third annual meeting of the ACL. Boston: Association for Computational Linguistics.

Kiss, Katalin E. 1998. Identificational focus versus information focus. Language 74: 245-273.

Larson, Richard. 1985. On the syntax of disjunction scope. Natural Language \& Linguistic Theory 3: 217-264.

Maekawa, Takafumi. 2004. Constituency, word order, and focus projection. In Proceedings of the HPSG04 Conference, ed. Stefan Müller. Stanford: CSLI.

Martin, Andrea, and Brian McElree. 2008. A content-addressable pointer mechanism underlies comprehension of verb phrase ellipsis. Journal of Memory and Language 58: 879-906.

Miller, George A., and Noam Chomsky. 1963. Finitary models of language users. In Vol. 2 of Handbook of mathematical psychology, eds. Luce R. Duncan, Robert R. Bush, and Eugene Galanter, 419-492. New York: Wiley.

Pollard, Carl, Robert Kasper, and Robert Levine. 1994. Studies in constituent ordering: toward a theory of linearization in Head-driven Phrase Structure Grammar. Research proposal to the National Science Foundation.

Reape, Michael. 1990. Getting things in order. Paper presented at 1990 Tilburg Conference on Syntactic Discontinuity. Slightly revised version published as Reape, 1996.

Reape, Michael. 1996. Getting things in order. In Discontinuous constituency, eds. Harry Bunt and Arthur van Horck, 209-257. Berlin: Mouton.

Romero, Maribel, and Chung-hye Han. 2003. Focus, ellipsis and the semantics of alternative questions. Empirical Issues in Formal Syntax 4: 291-307. 
Ross, John R. 1967. Constraints on variables in syntax. PhD Thesis, MIT.

Sag, Ivan A. 2010. Sign-based construction grammar: an informal synopsis. In Sign-based construction grammar, eds. Hans Boas and Ivan A. Sag. Stanford: CSLI.

Sag, Ivan A., Gerald Gazdar, Thomas Wasow, and Steven Weisler. 1985. Coordination and how to distinguish categories. Natural Language \& Linguistic Theory 3: 117-171.

Sag, Ivan A., Thomas Wasow, and Emily Bender. 2003. Syntactic theory: a formal introduction. Stanford: CSLI.

Schwarz, Bernhard. 1999. On the syntax of either ... or. Natural Language \& Linguistic Theory 17: 339370 .

Selkirk, Lisa. 1995. Sentence prosody: intonation, stress, and phrasing. In The handbook of phonological theory, ed. John Goldsmith, 550-569. London: Blackwell.

van Oirsouw, Robert R. 1987. The syntax of coordination. London: Croom Helm.

Wexler, Kenneth, and Peter Culicover. 1980. Formal principles of language acquisition. Cambridge: MIT Press.

Wilder, Chris. 1997. Some properties of ellipsis in coordination. In Studies on universal grammar and typological variation, eds. Alexiadou Artemis and T. Alan Hall. Amsterdam: John Benjamins.

Yatabe, Shuichi. 2001. Linearization-based syntax and semantics: an overview. Conference handbook 19: the nineteenth national conference of the English Linguistic Society in Japan, 192-197. 